\title{
Guía de práctica clínica para el tratamiento farmacológico y psicológico de los pacientes adultos con un trastorno del espectro esquizofrénico y un diagnóstico comórbido de trastorno por uso de sustancias
}

\section{Clinical practice guideline on pharmacological and psychological management of adult patients with schizophrenia spectrum disorders and a comorbid substance use}

\author{
Belén Arranz ${ }^{1 *}$, Marina Garriga ${ }^{1 * *}$, Miquel Bernardo**, Ana González-Pinto***, \\ Manuel Arrojo****, Marta Torrens*****, Judit Tirado-Muñoz*****, Francina Fonseca*****, \\ Pilar A. Sáiz******, Gerardo Flórez*******, José Manuel Goikolea**, Iñaki Zorrilla***, Ruth \\ Cunill*, Xavi Castells $* * * * * * * *$, Elisardo Becoña*********, Ana López*********, Luis San*. \\ ${ }^{1}$ Estos autores han contribuido por igual a este trabajo. \\ * Parc Sanitari Sant Joan de Deu, CIBERSAM. Universitat de Barcelona, Barcelona, España. \\ ** Hospital Clínic, Institut d'Investigacions Biomèdiques August Pi i Sunyer. Universitat de Barcelona, Barcelona, España. \\ *** Instituto de Investigación Sanitaria BIOARABA, OSI Araba, Hospital Universitario. CIBERSAM, Vitoria, España. \\ **** Servicio de Psiquiatría. EOXI de Santiago de Compostela, España. \\ ***** Institut de Neuropsiquiatria i Addiccions (INAD). Parc de Salut Mar, RTA, Barcelona, España. \\ ****** Universidad de Oviedo, CIBERSAM, Instituto de Investigación Sanitaria del Principado de Asturias (ISPA), Servicio de \\ Salud del Principado de Asturias (SESPA), Oviedo, España. \\ ******* Unidad de Conductas Adictivas, Complejo Hospitalario de Ourense. CIBERSAM, Ourense, España. \\ ******** Grupo de investigación TransLab, Departamento de Ciencias Médicas. Universitat de Girona, España. \\ ********* Unidad de Tabaquismo y Trastornos Adictivos, Facultad de Psicología. Univ. de Santiago de Compostela, España.
}

\section{Resumen}

Aunque el correcto diagnóstico y manejo de los pacientes con esquizofrenia y un diagnóstico comórbido de trastorno por uso de sustancias (TUS) determinaría una disminución de la morbilidad y mortalidad en estos pacientes, el desarrollo de estrategias terapéuticas eficientes es todavía una asignatura pendiente. Presentamos recomendaciones sobre el manejo farmacológico y psicológico de estos pacientes siguiendo la estructura PICO (Paciente-IntervenciónComparación-Outcome/resultados). Realizamos una evaluación de la calidad de los estudios y un resumen de la evidencia para cada pregunta siguiendo las recomendaciones del grupo de trabajo GRADE ( Grading of Recommendations, Assessment, Development and Evaluation»). Nuestros resultados sugieren: 1) En pacientes con esquizofrenia y trastorno por consumo de cannabis, no es posible recomendar un fármaco antipsicótico sobre otro (entre olanzapina, risperidona o haloperidol) para mejorar los síntomas psicóticos, reducir el consumo de cannabis o mejorar las variables pragmáticas

\begin{abstract}
Although correct diagnosis and management of patients with schizophrenia and a comorbid substance use disorder (SUD) would determine a decrease in morbidity and mortality in these patients, development of efficient therapeutic strategies is still pending. We present recommendations on the pharmacological and psychological management of these patients following the 'PICO' structure (Patient-Intervention-Comparison-Outcomes). Evaluation of the quality of studies and summary of the evidence for each question was performed following the recommendations of the GRADE (Grading of Recommendations, Assessment, Development and Evaluation) working group. Our results suggest: 1) In patients with schizophrenia and cannabis use disorder, it is not possible to recommend one antipsychotic drug over another (between olanzapine, risperidone or haloperidol) for improving psychotic symptoms, reducing cannabis use, or improving pragmatic variables (weak recommendation). Clozapine cannot be recommended to reduce cannabis use (weak
\end{abstract}

Recibido: Marzo 2020; Aceptado: Noviembre 2020.

Enviar correspondencia a:

Miguel Bernardo MD, PhD. Departamento de Psiquiatría, Hospital Clinic. Institut d'Investigacions Biomèdiques August Pi i Sunyer (IDIBAPS). Villarroel 170, 08036, Barcelona, España. 
(recomendación débil). No se puede recomendar la clozapina para reducir el consumo de cannabis (recomendación débil). 2) En pacientes con esquizofrenia y trastorno por consumo de cocaína, recomendamos haloperidol sobre olanzapina para reducir el craving (recomendación moderada) y olanzapina sobre haloperidol para mejorar los efectos secundarios motores en estos pacientes (recomendación moderada). 3) En pacientes con esquizofrenia y trastorno por consumo de alcohol, mientras que se recomienda naltrexona para reducir el consumo de alcohol (en términos de reducción del craving de alcohol) (recomendación débil), no hay evidencia suficiente para hacer ninguna recomendación sobre el uso de acamprosato como adyuvante (recomendación débil). 4) En pacientes con esquizofrenia y trastorno por consumo de nicotina, se recomiendan bupropión y vareniclina adyuvantes para reducir el consumo y la abstinencia de nicotina (recomendación fuerte/moderada). 5) En pacientes con esquizofrenia y trastorno por policonsumo, se recomiendan antipsicóticos de segunda generación sobre los de primera generación y olanzapina sobre otros antipsicóticos de segunda generación para mejorar los síntomas psicóticos (recomendación moderada/débil).

Palabras clave: Esquizofrenia; consumo de sustancias; comórbido; patología dual; antipsicótico; cannabis; cocaína; alcohol; nicotina. recommendation). 2) In patients with schizophrenia and cocaine use disorder we recommend haloperidol over olanzapine to reduce craving (moderate recommendation), and olanzapine over haloperidol to improve motor side effects in these patients (moderate recommendation). 3) In patients with schizophrenia and alcohol use disorder while naltrexone is recommended to reduce alcohol use (in terms of reducing alcohol craving) (weak recommendation), there is insufficient evidence to make any recommendation on the use of adjuvant acamprosate (weak recommendation). 4) In patients with schizophrenia and nicotine use disorder, adjuvant bupropion and varenicline are recommended for reducing nicotine use and nicotine abstinence (strong/moderate recommendation). 5) In patients with schizophrenia and polydrug use disorder, second-generation over first-generation antipsychotic drugs and olanzapine over other second-generation antipsychotics are recommended to improve psychotic symptoms (moderate/weak recommendation).

Keywords: Schizophrenia; substance use; comorbid; dual pathology; antipsychotic; cannabis; cocaine; alcohol; nicotine.

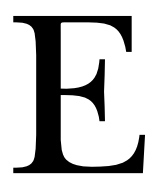

1 diagnóstico y tratamiento de las patologías duales, la coexistencia de un trastorno por uso de sustancias (TUS) en pacientes con enfermedad mental, plantea varios desafíos para los profesionales de la salud mental y los servicios de salud (Nielsen, Toftdahl, Nordentoft y Hjorthøj, 2017). En general, más del $25 \%$ de los pacientes en tratamiento en servicios de salud mental experimentan un TUS y casi el $70 \%$ de los pacientes en tratamiento en centros de adicción experimentan una enfermedad mental en algún momento de sus vidas (Hunt, Large, Cleary, Lai y Saunders, 2018).

Numerosos estudios han demostrado que el TUS es más frecuente en pacientes con esquizofrenia que en la población general (Addy, Radhakrishnan, Cortes y D’Souza, 2012; Fonseca-Pedrero, Lucas-Molina, Pérez-Albéniz, Inchausti y Ortuño-Sierra, 2020; Matali et al., 2016). La prevalencia general de TUS en personas con esquizofrenia es aproximadamente del 50\% (Regier et al., 1990; Thornton et al., 2012). La nicotina, el alcohol y el cannabis son las drogas más consumidas por los pacientes con esquizofrenia, seguidas de las anfetaminas en Australia y la cocaína en EE.UU. (García, Gomar, García-Portilla y Bobes, 2019; Green, Noordsy, Brunette y O'Keefe, 2008). En un subanálisis del estudio CATIE («Clinical Antipsychotics Trials of Intervention Effectiveness Project Schizophrenia Trial»), los porcentajes de consumo de sustancias para pacientes con esquizofrenia y un TUS comórbido fueron $87 \%$ para alcohol, $44 \%$ para cannabis y
$36 \%$ para cocaína (el consumo de nicotina no se contabilizó) (Swartz et al., 2008). El policonsumo es muy común; por ejemplo, los consumidores de cannabis también consumen nicotina y/o alcohol (Kavanagh, Mcgrath, Saunders, Dore y Clark, 2002) y se ha demostrado que el alcohol y el cannabis aumentan los efectos de la nicotina (Mueser y Gingerich, 2013). El uso de drogas de abuso en pacientes con esquizofrenia se asocia a un mayor riesgo de accidentes, conductas violentas, conductas autolesivas, peor pronóstico de psicosis, tasas más altas de hospitalización y uso de servicios psiquiátricos de emergencia, aumento de síntomas depresivos, conducta suicida, impulsividad, criminalidad y desempleo (Large, Mullin, Gupta, Harris y Nielssen, 2014; Heiberg et al., 2018). Además, las drogas de abuso pueden interactuar con los fármacos antipsicóticos, así afectando el perfil de efectos secundarios y la adherencia a la medicación (Margolese, Malchy, Negrete, Tempier y Gill, 2004).

Los pacientes con esquizofrenia y coexistencia de un TUS no suelen incluirse en los algoritmos tradicionales de tratamiento, aunque los árboles diferenciales de decisiones terapéuticas están disponibles para el consumo de drogas de abuso y esquizofrenia (Hasan et al., 2012, 2015). Además, los pacientes con esquizofrenia y un TUS comórbido suelen ser excluidos de los ensayos clínicos que evalúan la eficacia de diferentes fármacos psicoactivas, por temor a posibles interacciones entre las sustancias de abuso y el fármaco experimental, así como por la alta tasa de incumplimiento y 
abandono del tratamiento de esta población (Wobrock y Soyka, 2008).

Aunque el correcto diagnóstico y manejo de los pacientes con esquizofrenia con un TUS comórbido determinaría una disminución de la morbilidad y la mortalidad en estos pacientes, el desarrollo de estrategias terapéuticas eficientes aún es una asignatura pendiente (Addy et al., 2012). Con el desarrollo en las últimas dos décadas de enfoques de manejo integrados y planes de tratamiento individualizados (Crockford y Addington, 2017), la demanda de regímenes de tratamiento multidisciplinarios está aumentando, donde las intervenciones farmacológicas y psicológicas para el uso de sustancias y esquizofrenia se integran simultáneamente (Murthy, Mahadevan y Chand, 2019). Además, se recomienda un tratamiento combinado temprano tanto del TUS como de la esquizofrenia en pacientes con síntomas moderados o graves que afectan su funcionalidad (Hasan et al., 2012). Respecto a la duración del tratamiento, no hay evidencia que sugiera la necesidad de modificar los horarios temporales propuestos en los diferentes algoritmos terapéuticos para cada condición por separado (Hasan et al., 2015). La cuidadosa selección del tratamiento farmacológico que evalúe la eficacia, la seguri$\mathrm{dad}$, las posibles interacciones farmacológicas y los problemas relacionados con la adherencia es crucial en pacientes con una patología dual.

Hasta la actualidad, las revisiones y los metanálisis sistemáticos han informado los resultados de las intervenciones en pacientes con un TUS concurrente y un trastorno del espectro esquizofrénico (Baker, Thornton, Hiles, Hides y Lubman, 2012; Crockford y Addington, 2017; Hunt, Siegfried, Morley, Sitharthan y Cleary, 2013; Kishi y Iwata, 2015; Krause et al., 2019; Pearsall, Smith y Geddes, 2019; Temmingh, Williams, Siegfried y Stein, 2018; Wilson y Bhattacharyya, 2016). Que sepamos, sin embargo, no existe una guía de práctica clínica con recomendaciones claras sobre el manejo farmacológico y psicológico de estos pacientes. Por tanto, el objetivo de esta Guía de Práctica Clínica es proporcionar a los profesionales sanitarios (psiquiatras, profesionales del campo de la patología dual, psicólogos y médicos de atención primaria) implicados en la atención de pacientes con patología dual unas recomendaciones prácticas basadas en la evidencia científica para ayudar en el proceso de toma de decisiones en su práctica clínica. La guía también puede estar dirigida a otros profesionales en el campo de los TUS y a los pacientes y sus familias.

\section{Métodos}

\section{Creación del grupo de trabajo}

El grupo de trabajo multidisciplinar de elaboración de guías incluyó especialistas en psiquiatría, psicología y farmacología, con una amplia experiencia en el manejo de pacientes con diagnóstico dual. Se realizaron reuniones bimensuales entre mayo 2017 y mayo 2019 con el fin de manejar y analizar de forma independiente la evidencia recopilada de la literatura.

\section{Formulación de preguntas clínicas}

De acuerdo con los principios de la medicina basada en la evidencia, utilizamos la estructura PICO (Paciente-Intervención-Comparación-Outcome/resultados) (Guyatt et al., 2011) para formular la siguiente pregunta de revisión: "¿Cuál es el efecto de una intervención farmacológica y/o psicológica para el tratamiento de pacientes adultos con una enfermedad mental grave y un TUS?». La población objetivo de esta guía de práctica clínica era los pacientes mayores de 18 años diagnosticados con un trastorno del espectro esquizofrénico y un TUS (incluidos cannabis, cocaína, alcohol y/o nicotina). No se incluyó el trastorno por consumo de opioides porque no se encontraron revisiones sistemáticas con o sin metanálisis o ensayos clínicos aleatorizados.

El protocolo de estudio escrito se registró en la base de datos PROSPERO (CRD 42014013996).

\section{Búsqueda bibliográfica}

Realizamos una búsqueda exhaustiva de la literatura en MEDLINE, PsycINFO, Embase, Scopus, Web of Science, Cochrane Library y Pubmed hasta mayo de 2018. Se utilizaron los siguientes términos de búsqueda:

( ( ( “Schizophrenia”[Mesh] OR schizophrenia OR "Schizophrenia and Disorders with Psychotic Features"[Mesh])) AND ("Trifluoperazine"[Mesh] OR "Haloperidol"[Mesh] OR "Flupenthixol"[Mesh] OR "Perphenazine"[Mesh] OR "Chlorpromazine"[Mesh] OR "Methotrimeprazine"[Mesh] OR "levomepromazine maleate"[Supplementary Concept] OR first generation antipsychotic*)) AND (substance abuse OR substance dependence OR substance use OR comorbidity OR misuse OR co-occurr* OR coexist* OR concurren* OR dual diagnosis OR dual disorder OR dual pathology OR "Diagnosis, Dual (Psychiatry)"[Mesh])) AND ("Alcohol Drinking"[Mesh] OR "Drinking Behavior" [Mesh] OR "alcohol use" OR "alcohol abuse" OR "nicotine use" OR "Marijuana Abuse”[Mesh] OR "Marijuana Smoking" [Mesh] OR "cannabis use" OR "Cocaine-Related Disorders" [Mesh] OR "cocaine use" OR "cocaine abuse").

Límites: Review, Systematic Reviews, Meta-Analysis, Clinical Trial; Young Adult: 19-44 years; Middle Aged: 45-64 years.

( ( ("Schizophrenia"[Mesh] OR "Schizophrenia and Disorders with Psychotic Features"[Mesh])) AND (substance abuse OR substance dependence OR substance use OR comorbidity OR misuse OR co-occurr* OR coexist* OR concurren* OR dual diagnosis OR dual disorder OR dual pathology OR "Diagnosis, Dual (Psychiatry)" [Mesh])) AND ("Alcohol Drinking"[Mesh] OR "Drinking Behavior"[Mesh] OR "alcohol use" OR "alcohol abuse" OR "nicotine use" OR "Marijuana Abuse”[Mesh] 
OR "Marijuana Smoking"[Mesh] OR "cannabis use" OR "Cocaine-Related Disorders"[Mesh] OR "cocaine use" OR "cocaine abuse")) AND ("Risperidone"[Mesh] OR risperidone OR "olanzapine" [Supplementary Concept] OR "olanzapine fluoxetine combination" [Supplementary Concept] OR olanzapine OR "ziprasidone" [Supplementary Concept] OR ziprasidone OR "quetiapine" [Supplementary Concept] OR quetiapine OR "paliperidone palmitate" [Supplementary Concept] OR paliperidone OR "aripiprazole" [Supplementary Concept] OR aripiprazole OR "Asenapine" [Supplementary Concept] OR asenapine OR "zotepine" [Supplementary Concept] OR zotepine OR "sultopride" [Supplementary Concept] OR "sertindole" [Supplementary Concept] OR sertindole OR second generation antipsychotic*).

Límites: Review, Systematic Reviews, Meta-Analysis, Clinical Trial; Young Adult: 19-44 years; Middle Aged: 45-64 years.

( ((“"Schizophrenia”[Mesh] OR schizophrenia OR "Schizophrenia and Disorders with Psychotic Features"[Mesh])) AND (substance abuse OR substance dependence OR substance use OR comorbidity OR misuse OR co-occurr* OR coexist* OR concurren* OR dual diagnosis OR dual disorder OR dual pathology OR "Diagnosis, Dual (Psychiatry)"[Mesh])) AND ("Alcohol Drinking"[Mesh] OR "Drinking Behavior" [Mesh] OR "alcohol use" OR "alcohol abuse" OR "nicotine use" OR "Marijuana Abuse" [Mesh] OR "Marijuana Smoking" [Mesh] OR "cannabis use" OR "Cocaine-Related Disorders"[Mesh] OR "cocaine use" OR "cocaine abuse")) AND ("Lithium"[Mesh] OR "Lithium Chloride"[Mesh] OR "Lithium Carbonate"[Mesh] OR lithium OR "Valproic Acid"[Mesh] OR valproate OR "lamotrigine 2-Nglucuronide"[ Supplementary Concept] OR lamotrigine OR carbamazepine OR oxcarbazepine OR mood stabilizer*).

Límites: Review, Systematic Reviews, Meta-Analysis, Clinical Trial; Young Adult: 19-44 years; Middle Aged: 45-64 years.

( ((“"Schizophrenia”[Mesh] OR schizophrenia OR "Schizophrenia and Disorders with Psychotic Features"[Mesh])) AND (substance abuse OR substance dependence OR substance use OR comorbidity OR misuse OR co-occurr* OR coexist* OR concurren* OR dual diagnosis OR dual disorder OR dual pathology OR "Diagnosis, Dual (Psychiatry)"[Mesh])) AND ("Alcohol Drinking”[Mesh] OR "Drinking Behavior" [Mesh] OR "alcohol use" OR "alcohol abuse" OR "nicotine use" OR "Marijuana Abuse"[Mesh] OR "Marijuana Smoking"[Mesh] OR "cannabis use" OR "Cocaine-Related Disorders" [Mesh] OR "cocaine use" OR "cocaine abuse")) AND (("Disulfiram"[Mesh] OR disulfiram OR "Naltrexone" [Mesh] OR naltrexone OR "acamprosate" [Supplementary Concept] OR acamprosate OR "topiramate" [Supplementary Concept] OR topiramate OR "Bupropion"[Mesh] OR bupropion OR nicotine replace- ment therapy OR "varenicline" [Supplementary Concept] OR varenicline OR "varenicline $\mathrm{N}$-carbamoylglucuronide" [Supplementary Concept]) AND ((Clinical Trial[ptyp] OR Meta-Analysis[ptyp] OR Review[ptyp] OR systematic[sb] ) AND (adult[MeSH:noexp] OR aged[MeSH] ))).

Límites: Review, Systematic Reviews, Meta-Analysis, Clinical Trial; Young Adult: 19-44 years; Middle Aged: 45-64 years.

Los criterios de inclusión para los estudios publicados eran conformes a la estructura PICO (Guyatt et al., 2011): (a) diseño del estudio: metanálisis, revisión Cochrane, revisión sistemática, ensayo clínico aleatorizado doble o simple ciego; (b) población: sujetos con un trastorno relacionado con la esquizofrenia y consumo de cannabis, cocaína, alcohol o nicotina; (c) tratados con antipsicóticos de primera/ segunda generación / antipsicóticos atípicos / naltrexona / disulfiram / acamprosato / bupropión / vareniclina; (d) los resultados relacionados con la mejora de los síntomas de la esquizofrenia, y/o los resultados relacionados con el consumo de sustancias (disminución de uso/abstinencia), y/o variables pragmáticos, tales como efectos secundarios.

\section{Evaluación de la calidad de la evidencia y formulación de las recomendaciones}

Debido a la escasez de guías farmacológicas para este grupo de tratamiento, no se dispuso de un comparador consistente o de referencia. La evaluación de la calidad de los estudios y el resumen de la evidencia para cada pregunta se realizó siguiendo las recomendaciones del grupo de trabajo GRADE ( «Grading of Recommendations Assessment, Development and Evaluation») (www.gradeworkinggroup.org) (Schünemann et al., 2008).

El sistema GRADE asigna grados separados para la calidad de la evidencia y la fuerza de las recomendaciones (Mustafa et al., 2013). La calidad de la evidencia se define como la medida en que «uno puede estar seguro de que una estimación del efecto o asociación puede ser correcta». Esto se basa en la probabilidad de que una investigación más específica no cambie la confianza en la estimación. La fuerza de la recomendación indica «el grado de confianza del evaluador que el cumplimiento de la recomendación hará más bien que mal» (Gopalakrishna, Langendam, Scholten, Bossuyt y Leeflang, 2013). Las recomendaciones polémicas o carentes de evidencia se resolvieron por consenso del grupo de trabajo.

Dada la amplia variación en la metodología de los estudios, los resultados informados y el número limitado de informes de investigación originales centrados en cada antipsicótico, se decidió no realizar un análisis cuantitativo de los datos en forma de metaanálisis y, en cambio, limitar este informe a una síntesis cualitativa de toda la evidencia disponible. Cada artículo se leyó en detalle y se evaluó críticamente de acuerdo con GRADE, luego se discutió entre los autores, lo que resultó en una puntuación general de 
evaluación de calidad, posteriormente revisada por resultado individual. Todo el proceso culminó en una recomendación clínica. En aras de la claridad, las recomendaciones aquí se dividen por sustancia.

\section{Revisión y evaluación externas}

La guía fue revisada externamente por un grupo multidisciplinario e independiente de expertos seleccionados por sus conocimientos de la metodología de preparación de guías de práctica clínica, la patología abordada y el ámbito de aplicación. La versión final fue revisada y aprobada por el grupo de trabajo. La evidencia se evaluó utilizando el instrumento AGREE II ( «Appraisal of Guidelines for Research and Evaluation») (Makarski y Brouwers, 2014) (www.agreecollaboration.org), que contiene 23 ítems agrupados en 6 dominios: alcance y propósito, participación de las partes interesadas, rigor de desarrollo, claridad y presentación, apli- cabilidad e independencia editorial. Los ítems se califican de 1 (muy en desacuerdo) a 7 (muy de acuerdo).

\section{Resultados}

\section{Selección de estudios}

La Figura 1 describe el diagrama de flujo PRISMA que resulta en la selección del estudio. La búsqueda produjo 650 estudios. Se consideraron elegibles para una evaluación adicional 36 estudios. La selección definitiva incluyó 24 estudios. No se incluyeron en la Guía los ensayos abiertos, los estudios de cohortes o de casos y controles, los estudios transversales y observacionales, los informes de casos, las cartas, los carteles y los resúmenes de presentaciones en reuniones y conferencias de especialistas. Solo se incluyeron artículos publicados en inglés. Los datos se extrajeron de los estudios incluidos mediante una plantilla predefinida y la calidad de

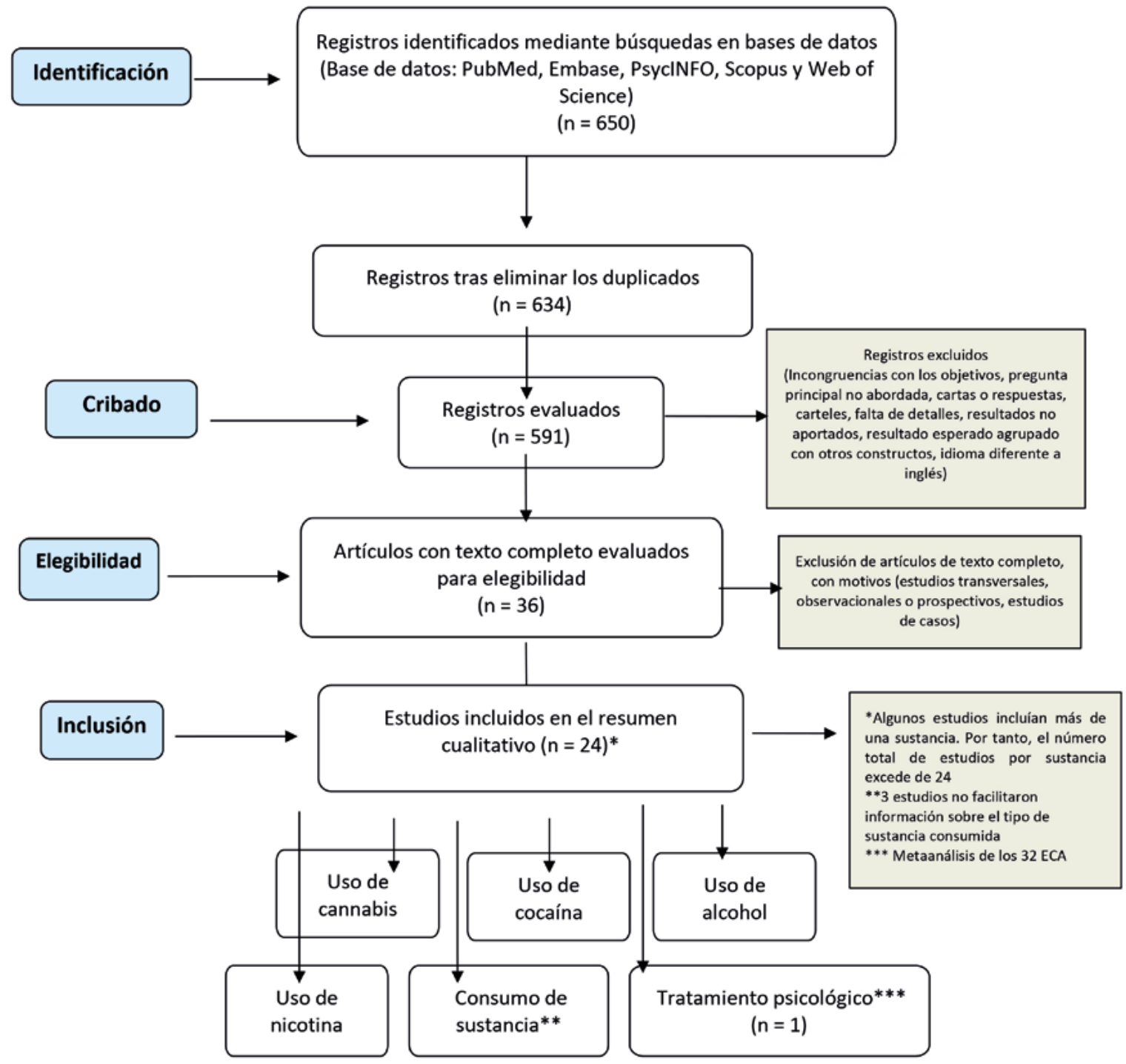

Figura 1. Diagrama de flujo del proceso de selección de estudios. 
Belén Arranz, Marina Garriga, Miquel Bernardo, Ana González-Pinto, Manuel Arrojo, Marta Torrens, Judit Tirado-Muñoz, Francina Fonseca, Pilar A. Sáiz, Gerardo Flórez, José Manuel Goikolea, Iñaki Zorrilla, Ruth Cunill, Xavi Castells, Elisardo Becoña, Ana López, Luis San

cada estudio se evaluó mediante criterios estándar. Las Tablas 1 a 5 presentan un informe resumido de estos estudios.

\section{Pacientes con esquizofrenia y trastorno por consumo de}

\section{cannabis}

Los detalles sobre los estudios incluidos se muestran en la Tabla 1.

Tabla 1. Estudios en esquizofrenia y trastorno por uso de cannabis.

\begin{tabular}{|c|c|c|c|c|c|c|c|}
\hline Autor & Diseño & Intervención & Pacientes & $\begin{array}{l}\operatorname{Exp}(n) / \\
\operatorname{Comp}(n)\end{array}$ & Seguimiento & $\begin{array}{c}\text { Variables de resultado } \\
\text { (clínicas, de consumo y } \\
\text { pragmáticas) }\end{array}$ & $\begin{array}{l}\text { Limitaciones } \\
\text { Sesgos }\end{array}$ \\
\hline $\begin{array}{l}\text { Akerele } \\
2007\end{array}$ & $\begin{array}{l}\text { ECA, } \\
\text { Doble-Ciego, } \\
\text { Ambulatorio }\end{array}$ & $\begin{array}{l}\text { 1. OLZ } 5-20 \mathrm{mg} / \mathrm{d} \\
\text { 2. RIS } 3-9 \mathrm{mg} / \mathrm{d}\end{array}$ & $\begin{array}{c}\text { Esquizofrenia } \\
\text { o TEA + TUS } \\
\text { (cocaína, cannabis). } \\
\text { SCID. }\end{array}$ & $10 / 13$ & $14 \mathrm{sem}$ & $\begin{array}{l}\text { - HAM-D. PANSS positiva, } \\
\text { PANSS negativa. } \\
\text { - Test de Orina, Craving. } \\
\text { - Efectos secundarios: } \\
\text { AIMS, SAS. }\end{array}$ & $\begin{array}{l}\text { Muestra pequeña. } \\
\text { Mayoritariamente } \\
\text { hombres ( } 89 \%) \text {. } \\
\text { Seguimiento corto. Posible } \\
\text { sesgo de publicación } \\
\text { selectiva. Financiado } \\
\text { por Lilly. Abandonos de } \\
\text { seguimiento: El 57,1\% } \\
\text { finalizó el estudio (OLZ } \\
n=6 ; \text { RIS } n=10) \text {. }\end{array}$ \\
\hline $\begin{array}{l}\text { Berk } \\
1999\end{array}$ & $\begin{array}{l}\text { ECA, } \\
\text { Doble-Ciego, } \\
\text { Hospitalizado }\end{array}$ & $\begin{array}{l}\text { 1. OLZ } 10 \mathrm{mg} / \mathrm{d} \\
\text { 2. } \mathrm{HAL} 10 \mathrm{mg} / \mathrm{d}\end{array}$ & $\begin{array}{l}\text { Psicosis + TUS } \\
\text { (cannabis) } \\
\text { MINI. }\end{array}$ & $15 / 15$ & $4 \mathrm{sem}$ & $\begin{array}{l}\text { - BPRS, CGI-S, CGI-I. } \\
\text { - Funcionamiento: GAF. } \\
\text { - Efectos secundarios: } \\
\text { SAS, BARS. }\end{array}$ & $\begin{array}{l}\text { Muestra pequeña. } \\
\text { Mayoritariamente hombres } \\
\text { (93,3\%). Seguimiento } \\
\text { corto. Periodo de lavado } \\
\text { de } 1 \text { día. El diagnóstico } \\
\text { de psicosis inducida por } \\
\text { cannabis es controvertido. }\end{array}$ \\
\hline $\begin{array}{l}\text { Brunette } \\
2011\end{array}$ & $\begin{array}{l}\text { ECA, } \\
\text { Simple-Ciego, } \\
\text { Bi-centrico, } \\
\text { Ambulatorio }\end{array}$ & $\begin{array}{l}\text { 1. Cambio a CLZ } \\
400-550 \mathrm{mg} / \mathrm{d} \\
\text { 2. Tratamiento } \\
\text { antipsicótico habitual }\end{array}$ & $\begin{array}{l}\text { Esquizofrenia } \\
\text { o TEA + TUS } \\
\text { (cannabis). } \\
\text { DSM-IV. }\end{array}$ & $15 / 16$ & $12 \mathrm{sem}$ & $\begin{array}{l}\text { - BPRS, CGI, SANS. } \\
\text { - Test de Orina, unidades } \\
\text { de consumo/semana, } \\
\text { Escala de abuso de } \\
\text { sustancia. } \\
\text { - SAS, BARS, AIMS, } \\
\text { adherencia al } \\
\text { tratamiento. }\end{array}$ & $\begin{array}{l}\text { Muestra pequeña. } \\
\text { Seguimiento corto. Periodo } \\
\text { de lavado de } 1 \text { día. Dosis } \\
\text { flexible de CLZ las primeras } \\
4 \text { semanas junto con } \\
\text { switch de antipsicótico } \\
\text { inicial. Posible sesgo de } \\
\text { publicación selectiva. }\end{array}$ \\
\hline $\begin{array}{l}\text { Green } \\
2004\end{array}$ & $\begin{array}{l}\text { ECA, } \\
\text { Doble-Ciego }\end{array}$ & $\begin{array}{l}\text { 1. OLZ } 5-20 \mathrm{mg} / \mathrm{d} \\
\text { 2. HAL } 2-20 \mathrm{mg} / \mathrm{d}\end{array}$ & $\begin{array}{c}\text { Primer episodio } \\
\text { psicótico } \\
\text { (Esquizofrenia, TEA, } \\
\text { Esquizofreniforme) } \\
\text { + TUS (cannabis, } \\
\text { alcohol). } \\
\text { DSM-IV, SCID. }\end{array}$ & $131 / 131$ & $12 \mathrm{sem}$ & - PANSS, CGI. & $\begin{array}{l}\text { Criterio exclusión: } \\
\text { dependencia de sustancias } \\
\text { en el último mes. Lilly } \\
\text { Research Laboratories } \\
\text { participó de forma } \\
\text { indirecta/directa en el } \\
\text { estudio. }\end{array}$ \\
\hline $\begin{array}{l}\text { Nimwegen } \\
2008\end{array}$ & $\begin{array}{l}\text { ECA, } \\
\text { Doble-Ciego, } \\
\text { Multicéntrico, } \\
\text { Hospitalizado + } \\
\text { Ambulatorio }\end{array}$ & $\begin{array}{l}\text { 1. OLZ } 5-20 \mathrm{mg} / \mathrm{d} \\
\text { 2. RIS } 1,25-5 \mathrm{mg} / \mathrm{d}\end{array}$ & $\begin{array}{l}\text { Esquizofrenia, } \\
\text { TEA, Esquizofreniform } \\
\text { + TUS (cannabis). } \\
\text { DSM-IV-TR, SCID. }\end{array}$ & $63 / 65$ & $6 \mathrm{sem}$ & $\begin{array}{l}\text { - OCDUS, DDQ, Cannabis } \\
\text { auto-reporte (reuniones } \\
\text { por semana). } \\
\text { - SWN. }\end{array}$ & $\begin{array}{l}\text { Muestra pequeña. Periodo } \\
\text { de seguimiento corto. } \\
\text { Solo } 41 / 128 \text { pacientes } \\
\text { consumían cannabis al } \\
\text { inicio del estudio. } 70 \% \text { de } \\
\text { los pacientes completaron } \\
\text { el estudio. Eli-Lilly participó } \\
\text { de forma indirecta/directa } \\
\text { en el estudio. }\end{array}$ \\
\hline $\begin{array}{l}\text { Sevy } \\
2011\end{array}$ & $\begin{array}{l}\text { ECA, } \\
\text { Simple-Ciego, } \\
\text { Hospitalizado }\end{array}$ & $\begin{array}{l}\text { 1. OLZ } 2,5-20 \mathrm{mg} / \mathrm{d} \\
\text { 2. RIS } 1-6 \mathrm{mg} / \mathrm{d}\end{array}$ & $\begin{array}{l}\text { Primer episodio de } \\
\text { Esquizofrenia, TEA, } \\
\text { Esquizofreniforme + } \\
\text { TUS (cannabis). }\end{array}$ & $28 / 21$ & $16 \mathrm{sem}$ & $\begin{array}{l}\text { - Respuesta al tratamiento, } \\
\text { síntomas positivos, CGI. } \\
\text { - Test de Orina, } \\
\text { Cuestionario de Uso } \\
\text { de Sustancias. } \\
\text { - Aumento de peso, SAS, } \\
\text { BARS. }\end{array}$ & $\begin{array}{l}\text { Muestra pequeña. El } \\
75 \% \text { de los pacientes del } \\
\text { grupo OLZ y el } 76 \% \text { del } \\
\text { grupo RIS completaron el } \\
\text { estudio. Posible sesgo de } \\
\text { publicación selectiva. }\end{array}$ \\
\hline $\begin{array}{l}\text { Siris } \\
1992\end{array}$ & $\begin{array}{l}\text { ECA, Doble- } \\
\text { Ciego. } \\
\text { Hospitalizado+ } \\
\text { Ambulatorio }\end{array}$ & $\begin{array}{l}\text { 1. IMI 50-200MG/día } \\
+ \text { trat. habitual } \\
\text { 2. Placebo + } \\
\text { tratamiento habitual }\end{array}$ & $\begin{array}{l}\text { Esquizofrenia o TEA+ } \\
\text { TUS (cannabis) } \\
\text { RDC. }\end{array}$ & $14 / 7$ & 9 sem & - CGI-I, CGI-S, SADS. & $\begin{array}{l}\text { Muestra pequeña. } \\
\text { Seguimiento corto. } \\
\text { La mayoría de tratamiento } \\
\text { habitual fue flufenazina } \\
\text { decanoato semanal. }\end{array}$ \\
\hline
\end{tabular}

Nota. AIMS: Abnormal Involuntary Movement Scale; BARS: Barnes Akathisia Rating Scale; BPRS: Brief Psychiatric Rating Scale; CGI: Clinical Global Impression, Impresión Clínica Global; CLZ: Clozapina; DDQ: Drug Desire Questionnaire; ECA: Ensayo clinico aleatorizado; GAF: Global Assessment Functioning; HAL: Haloperidol; HAM-D: Hamilton Depression Rating Scale; IMI: Imipramina; MINI: Mini International Neuropsychiatric Interview; OCDUS: Obsessive Compulsive Drug Use Scale; OLZ: Olanzapina; PANSS: Positive and Negative Syndrome Scale; RIS: Risperidona; SADS: Schedule for Affective Disorders and Schizophrenia; SANS: Scale for the Assessment of Negative Symptoms; SAS: Simpson Angus Scale; SCID: Structured Clinical Interview for DSM-IV; SWN: Subjective Well-being under Neuroleptics; Scale; TEA: Trastorno Esquizoafectivo;TUS: Trastorno por Uso de Sustancias. 
Pregunta 1 de PICO. ¿Es eficaz la administración de antipsicóticos para mejorar los síntomas de la esquizofrenia, reducir el consumo de cannabis o mejorar las variables pragmáticas y de funcionalidad en pacientes con esquizofrenia y consumo de cannabis?

Tres ensayos controlados aleatorios (ECA) evaluaron el efecto de los antipsicóticos para mejorar los síntomas de la esquizofrenia, olanzapina $v s$. risperidona en uno (Sevy et al., 2011) y olanzapina vs. haloperidol en dos ECA (Berk, Brook y Trandafir, 1999; Green et al., 2004). En la comparación de olanzapina y risperidona en pacientes hospitalizados con esquizofrenia o trastorno esquizoafectivo, no se encontraron diferencias en la mejoría de los síntomas positivos medidos con la escala SADS-C ni en el porcentaje de pacientes con respuesta clínica (evidencia de muy baja calidad) (Sevy et al., 2011). En los dos ECA que compararon olanzapina y haloperidol en pacientes hospitalizados con un primer episodio psicótico, esquizofrenia o trastorno esquizoafectivo, no se encontraron diferencias en el cambio medio de PANSS a la semana 12 ni en el porcentaje de pacientes con respuesta clínica (evidencia de muy baja calidad) (Green et al., 2004). Se observaron hallazgos similares en el cambio medio de las puntuaciones en la escala BPRS, puntuaciones en la escala CGI-S o puntuaciones en la escala CGI-I (evidencia de baja calidad) (Berk et al., 1999).

En los ECA realizados en pacientes con esquizofrenia o trastorno esquizoafectivo en entornos ambulatorios y de hospitalización, la comparación de olanzapina y risperidona para reducir el consumo de cannabis no mostró diferencias significativas usando diferentes medidas. Estos incluyeron análisis de orina de cannabis (evidencia de muy baja calidad) (Akerele y Levin, 2007); tres cuestionarios de craving (evidencia de calidad moderada) (Van Nimwegen et al., 2008); cuestionario sobre el craving de marihuana (evidencia de muy baja calidad) (Akerele y Levin, 2007); autoinformes (evidencia de calidad moderada) (Van Nimwegen et al., 2008); y cuestionario sobre consumo de sustancias (evidencia de muy baja calidad) (Sevy et al., 2011).

Respecto a las variables pragmáticas, olanzapina y risperidona no mostraron diferencias significativas en la escala SAS de efectos secundarios motores (evidencia de muy baja calidad) (Sevy et al., 2011); el índice de masa corporal (IMC) (evidencia de muy baja calidad) (Sevy et al., 2011); y la escala SWN (calidad de evidencia moderada) (Van Nimwegen et al., 2008).

\section{Recomendaciones}

- No es posible recomendar un fármaco antipsicótico sobre otro para mejorar los síntomas psicóticos, reducir el consumo de cannabis o mejorar las variables pragmáticas (recomendación débil).

Pregunta 2 de PICO. ¿Es eficaz la administración de antidepresivos adyuvantes para mejorar los sintomas de la esquizofrenia, reducir el consumo de cannabis o mejorar las variables pragmáti- cas y de funcionalidad en pacientes con esquizofrenia y consumo de cannabis?

Un ECA evaluó la imipramina adyuvante vs. placebo durante 9 semanas en pacientes con esquizofrenia o trastorno esquizoafectivo tratados con flufenazina (Siris, Bermanzohn, Mason, Rifkin y Alvir, 1992). El tratamiento con imipramina adyuvante se asoció con una disminución media de 0,54 y 0,93 puntos en las escalas CGI-S y CGI-I, respectivamente (evidencia de muy baja calidad). En la escala SADS, los pacientes tratados con imipramina mostraron disminuciones medias de 2,4, 6,9 y 7,1 puntos en los ítems «estado de ánimo», «otros síntomas asociados» y «síntomas endógenos», respectivamente, y un aumento medio de 0,26 puntos en el ítem «alucinaciones» en comparación con placebo, mientras que no se observaron diferencias en «ideación delirante» (evidencia de muy baja calidad). Además, los pacientes tratados con imipramina mostraron una disminución significativa de la puntuación media en la escala HDRS (evidencia de muy baja calidad).

\section{Recomendaciones}

- En pacientes con esquizofrenia y trastorno por consumo de cannabis concomitante, se recomienda imipramina adyuvante con el tratamiento antipsicótico actual para mejorar los síntomas afectivos (recomendación débil).

Pregunta 3 de PICO. ¿Es eficaz la administración de clozapina para mejorar los sintomas de la esquizofrenia, reducir el consumo de cannabis o mejorar las variables pragmáticas y de funcionalidad en pacientes con esquizofrenia y consumo de cannabis?

A pesar de la evidencia sobre la eficacia de la clozapina en pacientes con esquizofrenia y uso de sustancias (Arranz, Garriga, García-Rizo y San, 2018; Drake, Xie, McHugo y Green, 2000; Green, Zimmet, Strous y Schildkraut, 1999), solo un ECA ha evaluado el efecto de la clozapina en comparación con el tratamiento habitual para el uso de cannabis en pacientes ambulatorios con esquizofrenia y trastorno por consumo de cannabis concomitante (Brunette et al., 2011). En este estudio con un seguimiento semanal durante 12 semanas utilizando medidas de autoinforme, la clozapina no se asoció con una disminución significativa del consumo de cannabis (evidencia de muy baja calidad).

\section{Recomendaciones}

- En pacientes con esquizofrenia y trastorno por consumo de cannabis concomitante, no se puede recomendar el uso de clozapina para reducir el consumo de cannabis (recomendación débil).

\section{Pacientes con esquizofrenia y trastorno por consumo de cocaína}

Los detalles sobre los estudios incluidos se muestran en la Tabla 2. 
Belén Arranz, Marina Garriga, Miquel Bernardo, Ana González-Pinto, Manuel Arrojo, Marta Torrens, Judit Tirado-Muñoz, Francina Fonseca, Pilar A. Sáiz, Gerardo Flórez, José Manuel Goikolea, Iñaki Zorrilla, Ruth Cunill, Xavi Castells, Elisardo Becoña, Ana López, Luis San

Tabla 2. Estudios en esquizofrenia y trastorno por uso de cocaína.

\begin{tabular}{|c|c|c|c|c|c|c|c|}
\hline Autor & Diseño & Intervención & Pacientes & $\begin{array}{l}\operatorname{Exp}(n) / \\
\operatorname{Comp}(n)\end{array}$ & Seguimiento & $\begin{array}{c}\text { Variables de resultado } \\
\text { (clínicas, de consumo y } \\
\text { pragmáticas) }\end{array}$ & $\begin{array}{l}\text { Limitaciones } \\
\text { Sesgos }\end{array}$ \\
\hline $\begin{array}{l}\text { Akerele } \\
2007\end{array}$ & $\begin{array}{l}\text { ECA, } \\
\text { Doble-Ciego, } \\
\text { Ambulatorio }\end{array}$ & $\begin{array}{l}\text { 1. OLZ 5-20mg/d } \\
\text { 2. RIS } 3-9 \mathrm{mg} / \mathrm{d}\end{array}$ & $\begin{array}{l}\text { Esquizofrenia } \\
\text { o TEA+ TUS } \\
\text { (cocaína, } \\
\text { cannabis). } \\
\text { SCID. }\end{array}$ & $12 / 13$ & 14 sem & $\begin{array}{l}\text { - Hamilton-D. PANSS } \\
\text { positiva, PANSS negativa. } \\
\text { - Test de Orina, Craving } \\
\text { Puntuaciones. } \\
\text { - Efectos secundarios: } \\
\text { AIMS, SAS. Cumplimiento. }\end{array}$ & $\begin{array}{l}\text { Muestra pequeña. Seguimiento } \\
\text { corto. Mayoritariamente } \\
\text { hombres ( } 89 \%) \text {. Posible sesgo } \\
\text { de publicación selectiva. No se } \\
\text { recogieron datos de consumo } \\
\text { de otras sustancias. Financiado } \\
\text { por Eli Lilly. Abandonos de } \\
\text { seguimiento: El } 57,1 \% \text { finalizó el } \\
\text { estudio (OLZ n=6; RIS n=10). }\end{array}$ \\
\hline $\begin{array}{l}\text { Perry } \\
2004\end{array}$ & $\begin{array}{l}\text { ECA, } \\
\text { Doble-Ciego, } \\
\text { Hospitalario }\end{array}$ & $\begin{array}{l}\text { 1. Mazindol add-on } \\
+ \text { antipsicótico } \\
\text { habitual } \\
\text { 2. Placebo + } \\
\text { antipsicótico } \\
\text { habitual }\end{array}$ & $\begin{array}{l}\text { Esquizofrenia } \\
\text { o TEA+ TUS } \\
\text { (cocaína). } \\
\text { SCID. }\end{array}$ & $11 / 13$ & $6 \mathrm{sem}$ & $\begin{array}{l}\text { - PANSS positiva, PANSS } \\
\text { negativa. } \\
\text { - Test de Orina, Escala } \\
\text { visual analógica de craving, } \\
\text { Inventario cuantitativo de } \\
\text { Cocaína. } \\
\text { - Efectos secundarios: AIMS, } \\
\text { escala Webster modificada. }\end{array}$ & $\begin{array}{l}\text { Muestra pequeña. Seguimiento } \\
\text { corto. Posible sesgo de } \\
\text { publicación selectiva. } \\
\text { Antipsicóticos habituales } \\
\text { utilizados: } 9 \text { pacientes recibieron } \\
\text { HAL, } 5 \text { flufenacina, } 4 \text { PRZ } \\
\text { decanoato, } 1 \text { HAL decanoato, } 1 \\
\text { trifluoperazina, } 1 \text { CLZ y } 1 \text { RIS. }\end{array}$ \\
\hline $\begin{array}{l}\text { Sayers } \\
2005\end{array}$ & $\begin{array}{l}\text { ECA, } \\
\text { Doble-Ciego, } \\
\text { Ambulatorio }\end{array}$ & $\begin{array}{l}\text { 1. OLZ } 10 \mathrm{mg} / \mathrm{d} \\
\text { 2. } \mathrm{HAL} 10 \mathrm{mg} / \mathrm{d}\end{array}$ & $\begin{array}{l}\text { Esquizofrenia } \\
+ \text { TUS (cocaína). } \\
\text { DSM-IV. }\end{array}$ & $12 / 12$ & $26 \mathrm{sem}$ & $\begin{array}{l}\text { - BPRS, SANS, SAPS. } \\
\text { - Test de Orina. } \\
\text { Escala visual analógica. } \\
\text { - Efectos secundarios: } \\
\text { AIMS, BARS, SAS. }\end{array}$ & $\begin{array}{l}\text { De los } 170 \text { pacientes } \\
\text { inicialmente identificados, } \\
\text { solo se incluyeron } 24 \text { (muestra } \\
\text { pequeña). } \\
\text { Alta tasa de abandono. }\end{array}$ \\
\hline $\begin{array}{l}\text { Smelson } \\
2006\end{array}$ & $\begin{array}{l}\text { ECA, } \\
\text { Doble-Ciego }\end{array}$ & $\begin{array}{l}\text { 1. OLZ } 10 \mathrm{mg} / \mathrm{d} \\
\text { 2. HAL } 10 \mathrm{mg} / \mathrm{d} \\
\text { Las dosis fueron } \\
\text { de } 5 \text { a un máximo } \\
\text { de } 20 \mathrm{mg} / \mathrm{d}\end{array}$ & $\begin{array}{c}\text { Esquizofrenia } \\
+ \text { TUS (cocaína). } \\
\text { DSM-IV. }\end{array}$ & $16 / 15$ & $6 \mathrm{sem}$ & $\begin{array}{l}\text { - PANSS general, PANSS } \\
\text { positiva, PANSS negativa. } \\
\text { - Test de Orina. VCCQ. }\end{array}$ & $\begin{array}{l}\text { Muestra pequeña. Seguimiento } \\
\text { corto. Pacientes con otro } \\
\text { Trastornos del Eje I no fueron } \\
\text { incluidos. Posible sesgo de } \\
\text { enmascaramiento. Número de } \\
\text { abandonos: } 10 \mathrm{HAL}, 8 \text { OLZ. }\end{array}$ \\
\hline
\end{tabular}

Nota. AIMS: Abnormal Involuntary Movement Scale; BARS: Barnes Akathisia Rating Scale; BPRS: Brief Psychiatric Rating Scale; CLZ: Clozapina; ECA: Ensayo clinico aleatorizado; HAL: Haloperidol; HAM-D: Hamilton Depression Rating Scale; OLZ: Olanzapina; PANSS: Positive and Negative Syndrome Scale; RIS: Risperidona; SANS: Scale for the Assessment of Negative Symptoms; SAPS: Scale for the Assessment of Positive Symptoms; SAS: Simpson Angus Scale; SCID: Structured Clinical Interview for DSM-IV; TEA: Trastorno Esquizoafectivo; TUS: Trastorno por Uso de Sustancias; VCCQ: Voris Cocaine Craving Questionnaire.

Pregunta 4 de PICO. ¿Es eficaz la administración de antipsicóticos para mejorar los síntomas de la esquizofrenia, reducir el consumo de cocaina o mejorar las variables pragmáticas y de funcionalidad en pacientes con esquizofrenia y consumo de cocaína?

Dos ECA compararon olanzapina vs. haloperidol para mejorar los síntomas psicóticos en pacientes ambulatorios con esquizofrenia o trastorno esquizoafectivo (Sayers et al., 2005; Smelson et al., 2006). No se hallaron diferencias significativas a las 26 semanas de tratamiento utilizando una mejora del $30 \%$ en las escalas BPRS, SAPS o SANS. Tampoco se hallaron diferencias en los síntomas positivos PANSS, síntomas negativos PANSS y la subescala de síntomas generales PANSS (evidencia de baja calidad). Un ECA comparó olanzapina vs. Risperidona (Akerele y Levin, 2007) y no se observaron diferencias entre los grupos en las subescalas PANSS positivo y negativo.

Para el resultado del consumo de cocaína, olanzapina vs. haloperidol mostró resultados similares en dos ECA (Sayers et al., 2005; Smelson et al., 2006) que evaluaron el consumo de cocaína mediante prueba de drogas en orina con resultado positivo (evidencia de baja calidad). No se encontraron diferencias entre ambas drogas utilizando escalas de craving autoadministradas en los ítems de VCCQ «enfermo tras estímulo», «estado de ánimo tras estímulo» e «intensidad del craving tras estímulo» (Smelson et al., 2006). Sin embargo, la olanzapina se asoció con un mayor craving de cocaína en el ítem «energía después del estímulo» del VCCQ (Smelson et al., 2006) y la puntuación VAS de craving (Sayers et al., 2005) (evidencia de baja calidad). En un ECA que comparó olanzapina vs. risperidona, no se observaron diferencias entre estas dos drogas para reducir el consumo de cocaína (Akerele y Levin, 2007).

En relación a las variables pragmáticas, la olanzapina mostró significativamente menos efectos secundarios motores medidos con la escala AIMS que el haloperidol (Sayers et al., 2005) (evidencia de baja calidad).

\section{Recomendaciones}

- Se recomienda la administración de haloperidol sobre olanzapina para reducir el craving en pacientes con esquizofrenia y trastorno comórbido por consumo de cocaína (recomendación moderada).

- Se recomienda la administración de olanzapina sobre haloperidol para mejorar los efectos secundarios motores en pacientes con esquizofrenia y trastorno comórbido por consumo de cocaína (recomendación moderada). 
Pregunta 5 de PICO. ¿Es eficaz la administración de agonistas dopaminérgicos adyuvantes para mejorar los sintomas de la esquizofrenia, reducir el consumo de cocaína o mejorar las variables pragmáticas y de funcionalidad en pacientes con esquizofrenia y consumo de cocaína?

Un ECA evaluó la eficacia del tratamiento adyuvante con mazindol vs. placebo durante 6 semanas en 24 pacientes hospitalizados con esquizofrenia o trastorno esquizoafectivo (Perry et al., 2005). No se observaron diferencias entre los grupos de mazindol y placebo para todos los resultados, incluidos los síntomas positivos y negativos PANSS, el consumo de cocaína y la intensidad del craving medido con las escalas QCI y VAS autoadministrados, respectivamente, y la mejoría de los síntomas extrapiramidales medido con la escala AIMS o la escala de Webster modificada (evidencia de calidad moderada).

\section{Recomendaciones}

- No se puede recomendar el uso de agonistas dopaminérgicos para mejorar los síntomas psicóticos, reducir el consumo de cocaína o el craving de cocaína o mejorar las variables pragmáticas en los pacientes con esquizofrenia y trastorno comórbido de consumo de cocaína (recomendación débil).

\section{Pacientes con esquizofrenia y trastorno por consumo de alcohol}

Los detalles sobre los estudios incluidos se muestran en la Tabla 3.

Tabla 3. Estudios en esquizofrenia y trastorno por uso de alcohol.

\begin{tabular}{|c|c|c|c|c|c|c|c|}
\hline Autor & Diseño & Intervención & Pacientes & $\begin{array}{l}\operatorname{Exp}(n) / \\
\operatorname{Comp}(n)\end{array}$ & Seguimiento & $\begin{array}{l}\text { Variables de resultado } \\
\text { (clínicas, de consumo y } \\
\text { pragmáticas) }\end{array}$ & $\begin{array}{l}\text { Limitaciones } \\
\text { Sesgos }\end{array}$ \\
\hline $\begin{array}{l}\text { Green } \\
2004\end{array}$ & $\begin{array}{l}\text { ECA, } \\
\text { Doble-Ciego }\end{array}$ & $\begin{array}{l}\text { 1. OLZ 5-20mg/d } \\
\text { 2. HAL } 2-20 \mathrm{mg} / \mathrm{d}\end{array}$ & $\begin{array}{c}\text { Primer episodio } \\
\text { psicótico } \\
\text { (Esquizofrenia, } \\
\text { TEA, } \\
\text { Esquizofreniforme) } \\
\text { + TUS (cannabis, } \\
\text { alcohol). } \\
\text { DSM-IV, SCID. } \\
\end{array}$ & $131 / 131$ & $12 \mathrm{sem}$ & $\begin{array}{l}\text { - PANSS, CGI. Respuesta a } \\
\text { tratamiento. }\end{array}$ & $\begin{array}{l}\text { Criterio exclusión: dependencia } \\
\text { de sustancias en el último mes. } \\
\text { Lilly Research Laboratories } \\
\text { participó de forma indirecta/ } \\
\text { directa en el estudio. }\end{array}$ \\
\hline $\begin{array}{l}\text { Petrakis } \\
2004\end{array}$ & $\begin{array}{l}\text { ECA, } \\
\text { Doble-Ciego, } \\
\text { Ambulatorio }\end{array}$ & $\begin{array}{l}\text { 1. NTX 50mg/día } \\
\text { + tratamiento } \\
\text { habitual } \\
\text { 2. Placebo + } \\
\text { tratamiento } \\
\text { habitual }\end{array}$ & $\begin{array}{l}\text { Esquizofrenia/ } \\
\text { TEA + TUS } \\
\text { (alcohol). } \\
\text { SCID. }\end{array}$ & $16 / 15$ & $12 \mathrm{sem}$ & $\begin{array}{l}\text { - PANSS general, PANSS } \\
\text { positiva, PANSS negativa. } \\
\text { - Días de Consumo. Bebidas } \\
\text { por días de consumo. } \\
\text { Días de consumo abusivo. } \\
\text { TCQ. } \\
\text { - Efectos secundarios: } \\
\text { AIMS, HSCL. Adherencia. }\end{array}$ & $\begin{array}{l}\text { Muestra pequeña. Seguimiento } \\
\text { corto. Exclusivamente hombres } \\
(100 \%) \text {. Los participantes } \\
\text { también realizaron tratamiento } \\
\text { cognitivo conductual para } \\
\text { prevención de recaídas. } \\
\text { Pacientes compensados } \\
\text { económicamente (160\$). Cuatro } \\
\text { pacientes fueron hospitalizados } \\
\text { durante el estudio: dos del } \\
\text { grupo NTX y uno del placebo por } \\
\text { descompensación psicótica. }\end{array}$ \\
\hline $\begin{array}{l}\text { Ralevski } \\
2011\end{array}$ & $\begin{array}{l}\text { ECA, } \\
\text { Doble-Ciego, } \\
\text { Ambulatorio }\end{array}$ & $\begin{array}{c}\text { 1. ACAM } 1998 \mathrm{mg} / \\
\text { día + tratamiento } \\
\text { habitual } \\
\text { 2. Placebo + } \\
\text { tratamiento habitual }\end{array}$ & $\begin{array}{l}\text { Esquizofrenia, TEA, } \\
\text { Esquizofreniforme } \\
+ \text { TUS (alcohol). } \\
\text { DSM-IV, SCID. }\end{array}$ & $12 / 11$ & $12 \mathrm{sem}$ & $\begin{array}{l}\text { - PANSS, Hopkins Verbal } \\
\text { Learning Test, Gordon } \\
\text { Diagnostic System, WCST. } \\
\text { - Número de días de } \\
\text { consumo. Días de bebidas } \\
\text { fuertes. Bebidas por días } \\
\text { que bebe. Días de } \\
\text { abstinencia. OCDUS. } \\
\text { - Adherencia. }\end{array}$ & $\begin{array}{l}\text { Muestra pequeña. Seguimiento } \\
\text { corto. Mayoritariamente hombres } \\
(82,6 \% \text { ). Posible sesgo de } \\
\text { publicación selectiva. }\end{array}$ \\
\hline
\end{tabular}

Nota. ACAM: Acamprosato; AIMS: Abnormal Involuntary Movement Scale; ECA: Ensayo clinico aleatorizado; HAL: Haloperidol; HSCL: Hopkins Symptoms checklist; NTX: Naltrexona; OCDUS: Obsessive Compulsive Drug Use Scale; OLZ: Olanzapina; PANSS: Positive and Negative Syndrome Scale; SCID: Structured Clinical Interview for DSM-IV; TCQ: Tiffany Craving Questionnaire; TEA: Trastorno Esquizoafectivo; TUS: Trastorno por Uso de Sustancias; WCST: Wisconsin Card Sorting Test.

Pregunta 6 de PICO. ¿Es eficaz la administración de antipsicóticos para mejorar los síntomas de la esquizofrenia, reducir el consumo de alcohol o mejorar las variables pragmáticas y de funcionalidad en pacientes con esquizofrenia y consumo de alcohol?

Solo se publicó un ECA con 262 pacientes con un primer episodio de psicosis relacionado con la esquizofrenia y trastorno por consumo de alcohol concurrente para responder a esta pregunta (Green et al., 2004). Una comparación de olanzapina vs. haloperidol no mostró diferencias después de 12 semanas de tratamiento en la mejoría de la psicosis medida con cambios en PANSS o respuesta al tratamiento (PANSS y CGI) (evidencia de muy baja calidad).

\section{Recomendaciones}

- No hay evidencia suficiente para hacer cualquier recomendación respecto del uso de antipsicóticos para mejorar los síntomas psicóticos, reducir el consumo de alcohol y/o el craving de alcohol o mejorar las variables pragmáticas en los pacientes con esquizofrenia 
y trastorno comórbido de consumo de cocaína (recomendación débil).

Pregunta 7 de PICO. ¿Es eficaz la administración de antagonistas opiáceos (naltrexona) para mejorar los sintomas de la esquizofrenia, reducir el consumo de alcohol o mejorar las variables pragmáticas y de funcionalidad en pacientes con esquizofrenia y consumo de alcohol?

Un ECA evaluó el uso adyuvante de naltrexona vs. placebo en 31 pacientes ambulatorios con esquizofrenia o trastorno esquizoafectivo (Petrakis et al., 2004). A las 12 semanas de tratamiento, no se encontraron diferencias en las subescalas PANSS ni en consumo de alcohol (reducción del número de días del consumo de alcohol, días de consumo excesivo y el número de bebidas por día de consumo de alcohol) (evidencia de muy baja calidad). En la evaluación del craving de alcohol con el instrumento TCQ, los pacientes del grupo de naltrexona mostraron una reducción media de 2,17 puntos (disminución del craving) (evidencia de muy baja calidad).

\section{Recomendaciones}

- En pacientes con esquizofrenia y trastorno por consumo de alcohol concurrente, se recomienda la naltrexona para reducir el consumo de alcohol (en términos de reducción del craving de alcohol) (recomendación débil).

Pregunta 8 de PICO. ¿Es eficaz la administración de acamprosato adyuvante para mejorar los sintomas de la esquizofrenia, reducir el consumo de alcohol o mejorar las variables pragmáticas y de funcionalidad en pacientes con esquizofrenia y consumo de alcohol?

Un ECA evaluó el uso de acamprosato vs. placebo en 23 pacientes ambulatorios con un trastorno del espectro esquizofrénico y dependencia del alcohol (Ralevski et al., 2011). Después de 12 semanas de tratamiento, una batería de pruebas neuropsicológicas no encontró una mejora en la función cognitiva. En el cuestionario Hopkins de $30 \mathrm{mi}-$ nutos de retraso, el acamprosato obtuvo una puntuación significativamente mejor que el placebo, pero en las pruebas de distracción Gordon Box y las pruebas de porcentaje de errores perseverativos de Wisconsin, el grupo de acamprosato obtuvo una puntuación significativamente peor en comparación con el placebo (evidencia de muy baja calidad). En el análisis del resultado del consumo de alcohol, el acamprosato adyuvante no fue superior al placebo en número de días de consumo de alcohol, días de consumo excesivo de alcohol, número de bebidas por día de consumo de alcohol, días consecutivos de abstinencia y craving de alcohol (evidencia de baja o muy baja calidad).

\section{Recomendaciones}

- No hay evidencia suficiente para recomendar el uso de acamprosato adyuvante para mejorar los síntomas psicóticos, reducir el consumo de alcohol o mejorar las variables pragmáticas en pacientes con esquizofre- nia con trastorno por consumo de alcohol comórbido (recomendación débil).

\section{Pacientes con esquizofrenia y trastorno por consumo de nicotina}

Los detalles sobre los estudios incluidos se muestran en la Tabla 4.

Pregunta 9 de PICO. ¿Es eficaz la administración de bupropión adyuvante para mejorar los sintomas de la esquizofrenia, reducir el uso de nicotina o mejorar las variables pragmáticas y funcionales en pacientes con esquizofrenia y uso de nicotina?

Tres ECA evaluaron el efecto del tratamiento de 12 semanas con bupropión adyuvante vs. placebo en pacientes ambulatorios con esquizofrenia (Evins, Mays, Rigotti, Tisdale, Cather y Goff, 2001); Evins et al., 2007; George et al., 2002). No se encontraron diferencias entre bupropión y placebo respecto a la mejora de los síntomas positivos y negativos de las subescalas PANSS y el cuestionario de Ham-D (evidencia de baja calidad).

Cinco ECA evaluaron el resultado de la abstinencia de nicotina a los seis meses de seguimiento para las comparaciones de bupropión vs. placebo y bupropión y parche transdérmico de nicotina vs. placebo y parche transdérmico de nicotina (Evins et al., 2001, 2005, 2007; George et al., 2002, 2008). No se encontraron diferencias en los estudios individuales, pero el análisis de los datos de los cinco ECA mostró tasas de abstinencia casi tres veces más altas en los grupos de bupropión. En seis ECA en los que el resultado fue la abstinencia de nicotina al final del período de tratamiento activo (Evins et al., 2001, 2005, 2007; George et al., 2002, 2008), el bupropión fue significativamente más eficaz que el placebo, pero esta diferencia no se observó en la comparación de bupropión y parche transdérmico de nicotina vs. placebo y parche transdérmico de nicotina (evidencia de muy baja calidad). El análisis de los datos agrupados de los siete ECA mostró diferencias significativas a favor del bupropión.

Cuando la abstinencia de fumar se determinó mediante el nivel de $\mathrm{CO}$ en el aire espirado a los seis meses de seguimiento (Evins et al., 2001, 2005, 2007), los niveles de CO espirado en comparación con el valor inicial disminuyeron significativamente en el grupo de bupropión (evidencia de calidad moderada). Cuando se determinaron los niveles de CO espirado al final del período de tratamiento activo (Evins et al., 2001, 2005, 2007; Weiner et al., 2012), también se observaron diferencias a favor de bupropión (evidencia de calidad moderada). En tres ECA que evaluaron la abstinencia autoinformada de tabaquismo al final de un período de tratamiento de 12 semanas (Evins et al., 2001, 2005, 2007) se encontraron diferencias significativas en el grupo de bupropión vs. placebo (evidencia de calidad moderada). En relación a la reducción del número de cigarrillos/día al final del tratamiento de ocho semanas en pacientes hospi- 
Tabla 4. Estudios en esquizofrenia y trastorno por uso de nicotina.

BUPROPION VS PLACEBO

\begin{tabular}{|c|c|c|c|c|c|c|c|}
\hline Autor & Diseño & Intervención & Pacientes & Seguimiento & $\begin{array}{c}\text { Terapias } \\
\text { concomitantes }\end{array}$ & $\begin{array}{l}\text { Variables de resultado } \\
\text { (clínicas, de consumo y } \\
\text { pragmáticas) }\end{array}$ & $\begin{array}{l}\text { Limitaciones } \\
\text { Sesgos }\end{array}$ \\
\hline $\begin{array}{l}\text { Akbarpour } \\
2010\end{array}$ & $\begin{array}{l}\text { ECA, } \\
\text { Hospitalizados }\end{array}$ & $\begin{array}{l}\text { Bupropion } \\
300 \mathrm{mg} / \mathrm{d} \\
\text { Placebo }\end{array}$ & $\begin{array}{l}32 \text { pacientes } \\
\text { Solo hombres }\end{array}$ & $8 \mathrm{sem}$ & $\begin{array}{l}\text { No se efectuó } \\
\text { ninguna otra } \\
\text { intervención. }\end{array}$ & $\begin{array}{l}\text {-Abstinencia: no se determinó } \\
\text {-Disminución del consumo: número de } \\
\text { cigarrillos. No confirmación biológica. } \\
\text {-Estado mental: MMSE. }\end{array}$ & $\begin{array}{l}\text { Solo se incluyeron } \\
\text { hombres. } \\
\text { No se proporciona } \\
\text { información sobre } \\
\text { el tratamiento } \\
\text { farmacológico de } \\
\text { los pacientes. } \\
\text { No confirmación } \\
\text { biológica de la } \\
\text { disminución del } \\
\text { consumo. }\end{array}$ \\
\hline $\begin{array}{l}\text { Bloch } \\
2010\end{array}$ & $\begin{array}{l}\text { ECA, } \\
\text { Ambulatorio }\end{array}$ & $\begin{array}{l}\text { Bupropion } \\
300 \mathrm{mg} / \mathrm{d} \\
\text { Placebo }\end{array}$ & $\begin{array}{l}61 \text { pacientes } \\
46 \text { hombres }\end{array}$ & 14 sem & $\begin{array}{l}\text { Ambos grupos } \\
\text { recibieron } 15 \\
\text { sesiones de TCC. }\end{array}$ & $\begin{array}{l}\text {-Abstinencia: no se determinó } \\
\text {-Disminución del consumo: número de } \\
\text { cigarrillos, test de Fargestrom en la sem } 7 \\
\text { y } 14 . \\
\text {-Estado mental: PANSS y BPRS. }\end{array}$ & $\begin{array}{l}\text { No se proporciona } \\
\text { información sobre } \\
\text { el tratamiento } \\
\text { farmacológico de } \\
\text { los pacientes. }\end{array}$ \\
\hline $\begin{array}{l}\text { Evins } \\
2001\end{array}$ & $\begin{array}{l}\mathrm{ECA}, \\
\text { Ambulatorio }\end{array}$ & $\begin{array}{l}\text { Bupropion } \\
150 \mathrm{mg} / \mathrm{d} \\
\text { Placebo }\end{array}$ & $\begin{array}{l}19 \text { pacientes. } \\
\text { Dosis de } \\
\text { antipsicótico } \\
\text { estable. } \\
8 \text { pacientes } \\
\text { con CLZ }\end{array}$ & $\begin{array}{l}12 \text { sem } \\
\text { tratamiento } \\
\text { activo } \\
6 \text { m de } \\
\text { seguimiento }\end{array}$ & $\begin{array}{l}\text { Ambos } \\
\text { grupos } \\
\text { recibieron } 9 \\
\text { sesiones de } \\
\text { TCC de } 1 \text { hora. }\end{array}$ & $\begin{array}{l}\text {-Abstinencia a la semana } 12 \text { y } 24 \text { (auto- } \\
\text { informes verificados mediante niveles CO } \\
\text { aire expirado < } 9 \text { ppm o cotinina en suero < } \\
14 \mathrm{ng} / \mathrm{ml} \text { ). } \\
\text {-Disminución del número de cigarrillos, } \\
\text { determinado mediante cotinina sérica, } \\
\text { reducción del } 50 \% \text { del número de cigarrillos } \\
\text { y disminución del } 30 \% \text { en CO expirado. } \\
\text {-Estado mental: BPRS, SANS y HAM-D. } \\
\text {-Síntomas extrapiramidales: SAS y AIMS. }\end{array}$ & $\begin{array}{l}\text { Un paciente se } \\
\text { retiró del estudio } \\
\text { antes de iniciar } \\
\text { tratamiento. }\end{array}$ \\
\hline $\begin{array}{l}\text { Evins } \\
2005\end{array}$ & $\begin{array}{l}\text { ECA, } \\
\text { Ambulatorio }\end{array}$ & $\begin{array}{l}\text { Bupropion } \\
300 \mathrm{mg} / \mathrm{d} \\
\text { Placebo }\end{array}$ & $\begin{array}{l}57 \text { pacientes. } \\
39 \text { hombres. } \\
12 \text { con CLZ }\end{array}$ & $\begin{array}{l}12 \text { sem } \\
\text { tratamiento } \\
\text { activo } \\
6 \text { m de } \\
\text { seguimiento }\end{array}$ & $\begin{array}{l}\text { Ambos grupos } \\
\text { recibieron } 12 \\
\text { sesiones de } \\
\text { TCC de } 1 \text { hora. }\end{array}$ & $\begin{array}{l}\text {-Abstinencia a los } 7 \text { días, y a las } 4,12 \text { y } \\
24 \text { semanas (auto-informes verificados } \\
\text { mediante niveles CO aire expirado }<9 \text { ppm. } \\
\text {-Disminución del número de cigarrillos } \\
\text { mediante el CO expirado y auto-informes. } \\
\text {-Estado mental: PANSS, SANS, HAM-D y } \\
\text { HAM-A. } \\
\text {-Parkinsonismo: SAS y AIMS. }\end{array}$ & $\begin{array}{l}\text { En el grupo } \\
\text { placebo se } \\
\text { aleatorizaron } \\
\text { mayor número } \\
\text { de pacientes con } \\
\text { clozapina }(11 / 28) \\
\text { que en el grupo } \\
\text { bupropion }(1 / 25) \text {. }\end{array}$ \\
\hline $\begin{array}{l}\text { Evins } \\
2007\end{array}$ & $\begin{array}{l}\text { ECA, } \\
\text { Ambulatorio }\end{array}$ & $\begin{array}{l}\text { Bupropion } \\
300 \mathrm{mg} / \mathrm{d} \\
\text { Placebo }\end{array}$ & $\begin{array}{l}51 \text { pacientes } \\
16 \text { con } C L Z\end{array}$ & $\begin{array}{l}12 \text { sem } \\
\text { tratamiento } \\
\text { activo } \\
6 \text { m de } \\
\text { seguimiento }\end{array}$ & $\begin{array}{l}\text { Ambos grupos } \\
\text { recibieron: } \\
\text { (1) } 12 \text { sesiones } \\
\text { de TCC de } 1 \\
\text { hora; (2) Parche } \\
\text { transdérmico a } \\
\text { dosis } \\
\text { decrecientes } \\
\text { (3) chicle de } \\
\text { nicotina en caso } \\
\text { necesario. }\end{array}$ & $\begin{array}{l}\text {-Abstinencia a la sem } 8,12,24 \text { y } 52 \\
\text { mediante auto-informe y comprobada } \\
\text { mediante CO expirado < } 8 \text { ppm. } \\
\text {-Disminución número cigarrillos en la sem } \\
12 \text { y } 24 \text {. } \\
\text {-Estado mental: PANSS, SANS, HAM-D } \\
\text { y STAI. } \\
\text { - Parkinsonismo: SAS y AIMS. }\end{array}$ & $\begin{array}{l}5 / 25 \text { en grupo } \\
\text { bupropion y } 8 / 26 \\
\text { en grupo control } \\
\text { abandonaron } \\
\text { el estudio. La } \\
\text { distribución por } \\
\text { sexos no queda } \\
\text { clara. }\end{array}$ \\
\hline $\begin{array}{l}\text { George } \\
2002\end{array}$ & $\begin{array}{l}\mathrm{ECA}, \\
\text { Ambulatorio }\end{array}$ & $\begin{array}{l}\text { Bupropion } \\
300 \mathrm{mg} / \mathrm{d} \\
\text { Placebo }\end{array}$ & $\begin{array}{l}32 \text { pacientes } \\
18 \text { hombres }\end{array}$ & $\begin{array}{l}10 \text { sem } \\
\text { tratamiento } \\
\text { activo } \\
6 \text { m de } \\
\text { seguimiento }\end{array}$ & $\begin{array}{l}\text { Ambos grupos } \\
\text { recibieron } 10 \\
\text { sesiones de } \\
\text { terapia grupal } \\
\text { motivacional, } \\
\text { psicoeducación y } \\
\text { prevenció } \\
\text { de recaídas. }\end{array}$ & $\begin{array}{l}\text {-Abstinencia en semana } 10 \text { y en mes } 6 \text { (CO } \\
\text { expirado <10ppm) } \\
\text { - Disminución del consumo (auto-informes } \\
\text { y CO expirado) } \\
\text {-Estado mental: PANSS y BDI. } \\
\text {-Parkinsonismo: Escala Webster y AIMS. }\end{array}$ & No se evidencian. \\
\hline $\begin{array}{l}\text { George } \\
2008\end{array}$ & $\begin{array}{l}\text { ECA, } \\
\text { Ambulatorio }\end{array}$ & $\begin{array}{l}\text { Bupropion } \\
300 \mathrm{mg} / \mathrm{d} \\
\text { Placebo }\end{array}$ & $\begin{array}{l}59 \text { pacientes } \\
35 \text { hombres } \\
9 \text { con CLZ }\end{array}$ & $\begin{array}{l}10 \text { sem } \\
\text { tratamiento } \\
\text { activo } \\
6 \text { m de } \\
\text { seguimiento }\end{array}$ & $\begin{array}{l}\text { Ambos grupos } \\
\text { recibieron } 10 \\
\text { sesiones de } \\
\text { terapia grupal } \\
\text { conductual } \\
\text { y parche } \\
\text { transdérmico. }\end{array}$ & $\begin{array}{l}\text {-Abstinencia entre los días } 43 \text { y } 70 \\
\text { y a los } 6 \text { m (auto-informes y CO expirado } \\
\text { <10ppm). } \\
\text {-La disminución del consumo no fue una } \\
\text { variable de resultado } \\
\text {-Estado mental: PANSS, BDI y HAM-D. }\end{array}$ & No se evidencian. \\
\hline $\begin{array}{l}\text { Weiner } \\
2012\end{array}$ & $\begin{array}{l}\text { ECA, } \\
\text { Ambulatorio }\end{array}$ & $\begin{array}{l}\text { Bupropion } \\
300 \mathrm{mg} / \mathrm{d} \\
\text { Placebo }\end{array}$ & $\begin{array}{l}46 \text { pacientes } \\
37 \text { hombres } \\
13 \text { con } \mathrm{CLZ} \\
28 \text { con } \mathrm{AP} \\
\text { atípicos }\end{array}$ & 12 sem & $\begin{array}{l}\text { Ambos grupos } \\
\text { recibieron } 9 \\
\text { sesiones de } \\
\text { terapia grupal. }\end{array}$ & $\begin{array}{l}\text {-Abstinencia: CO expirado<10 ppm en } 4 \\
\text { visitas } \\
\text {-Disminución del consumo: CO expirado, } \\
\text { Test Fargestrom y cotinina en orina } \\
\text {-Estado mental: BPRS y SANS } \\
\text {-Efectos secundarios motores: SAS } \\
\text {-Variables neuropsicológicas. }\end{array}$ & No se evidencian. \\
\hline
\end{tabular}


VARENICLINA VS PLACEBO

\begin{tabular}{|c|c|c|c|c|c|c|c|}
\hline Autor & Diseño & $\begin{array}{c}\text { Grupos de } \\
\text { Intervención }\end{array}$ & Pacientes & Seguimiento & $\begin{array}{c}\text { Terapias } \\
\text { concomitantes }\end{array}$ & $\begin{array}{l}\text { Variables de resultado } \\
\text { (clínicas, de consumo y } \\
\text { pragmáticas) }\end{array}$ & $\begin{array}{l}\text { Limitaciones } \\
\text { Sesgos }\end{array}$ \\
\hline $\begin{array}{l}\text { Williams } \\
2012\end{array}$ & $\begin{array}{l}\text { ECA, } \\
\text { Ambulatorio }\end{array}$ & $\begin{array}{c}\text { Vareniclina } 1 \\
\mathrm{mg} / \mathrm{d} \\
\text { Placebo }\end{array}$ & $\begin{array}{l}128 \text { pacientes } \\
98 \text { hombres } \\
109 \text { con AP } \\
\text { atípicos }\end{array}$ & $12 \mathrm{sem}$ & $\begin{array}{l}\text { Ambos grupos } \\
\text { recibieron apoyo } \\
\text { psicológico. }\end{array}$ & $\begin{array}{l}\text {-Abstinencia a la semana 4, } 12 \text { y } 24 \text { (auto- } \\
\text { informes verificados mediante niveles CO } \\
\text { aire expirado < } 10 \text { ppm). } \\
\text {-Disminución del consumo: disminución del } \\
50 \% \text { en el número de cigarrillos. } \\
\text {-Estado mental: PANSS, SAS, CSSRS, CGI. } \\
\text {-Síntomas extrapiramidales: SAS y AIMS. }\end{array}$ & $\begin{array}{l}\text { Ratio } 2: 1 \\
\text { (vareniclina: } \\
\text { placebo). }\end{array}$ \\
\hline $\begin{array}{l}\text { Weiner } \\
2011\end{array}$ & $\begin{array}{l}\text { ECA, } \\
\text { Ambulatorio }\end{array}$ & $\begin{array}{l}\text { Vareniclina } 1 \\
\mathrm{mg} / \mathrm{d} \\
\text { Placebo }\end{array}$ & $\begin{array}{l}9 \text { pacientes } \\
\text { Todos con AP } \\
\text { atípicos }\end{array}$ & $12 \mathrm{sem}$ & $\begin{array}{l}\text { Terapia } \\
\text { psicológica } \\
\text { individual. }\end{array}$ & $\begin{array}{l}\text {-Abstinencia: CO expirado<10 ppm en sem } \\
12 . \\
\text {-Disminución del consumo: CO expirado. } \\
\text {-Estado mental: BPRS y CDS. }\end{array}$ & $\begin{array}{l}\text { No se incluyen } \\
\text { datos demográficos } \\
\text { de los pacientes. }\end{array}$ \\
\hline
\end{tabular}

Nota. AIMS: Abnormal Involuntary Movement Scale; AP: Antipsicóticos; BDI: Beck Depression Inventory; BPRS: Brief Psychiatric Rating Scale; CGI: Clinical Global Impression, Impresión Clínica Global; CLZ: Clozapina; CO: Monóxido de carbono; CDS: Calgary Depression Scale; CSSRS: Columbia Suicide Severity Rating Scale; ECA: Ensayo clinico aleatorizado; GAF: Global Assessment Functioning; HAM-A: Hamilton Anxiety Rating Scale; HAM-D: Hamilton Depression Rating Scale; MMSE: MiniMental State Examination; PANSS: Positive and Negative Syndrome Scale; SANS: Scale for the Assessment of Negative Symptoms; SAS: Simpson Angus Scale; State Trait Anxiety Inventory (STAI), TCC: Terapia Cognitivo-conductual.

talizados (Akbarpour et al., 2010) o del tratamiento de 14 semanas en pacientes ambulatorios (Bloch et al., 2010), no se encontraron diferencias entre bupropión y placebo (evidencia de baja calidad).

\section{Recomendaciones}

- La evidencia es insuficiente para hacer una recomendación sobre el uso de bupropión para reducir los síntomas psicóticos (recomendación débil).

- Se recomienda bupropión adyuvante para reducir el uso de nicotina y la abstinencia de nicotina en pacientes con esquizofrenia y dependencia de nicotina concurrente (recomendación fuerte/moderada).

Pregunta 10 de PICO. ¿Es eficaz la administración de vareniclina adyuvante para mejorar los sintomas de la esquizofrenia, reducir el uso de nicotina o mejorar las variables pragmáticas y funcionales en pacientes con esquizofrenia y uso de nicotina?

Dos ECA evaluaron el uso de vareniclina adyuvante $v s$. placebo después de 12 semanas de tratamiento en pacientes ambulatorios con esquizofrenia utilizando los niveles de CO espirado y la abstinencia autoinformada de tabaquismo (Weiner et al., 2011; Williams et al., 2012). La vareniclina fue significativamente más efectiva que el placebo para lograr la abstinencia a las 12 semanas (evidencia de calidad moderada) pero las diferencias desaparecieron a los seis meses de seguimiento.

\section{Recomendaciones}

- No hay evidencia de la eficacia de la vareniclina para reducir los síntomas psicóticos.

- Se recomienda la vareniclina adyuvante para lograr la abstinencia de nicotina en pacientes con esquizofrenia y dependencia a la nicotina concurrente (recomendación fuerte/moderada).

\section{Pacientes con esquizofrenia y trastorno por policonsumo}

Los detalles sobre los estudios incluidos se muestran en la Tabla 5 .
Pregunta 11 de PICO. ¿Es eficaz la administración de antipsicóticos para mejorar los síntomas de la esquizofrenia, reducir el consumo general de sustancias o mejorar las variables pragmáticas y de funcionalidad en pacientes con esquizofrenia y policonsumo de drogas?

Tres ECA compararon olanzapina vs. risperidona (Akerele y Levin, 2007; Green et al., 2004) y cinco brazos de tratamiento con antipsicóticos (olanzapina vs. perfenazina vs. risperidona vs. quetiapina vs. ziprasidona) (Swartz et al., 2008) en pacientes hospitalizados y ambulatorios con esquizofrenia. En el resultado de mejora de los síntomas psicóticos, la olanzapina fue significativamente más eficaz que la risperidona a los 18 meses en todas las comparaciones utilizando CGI-S, puntuación total PANSS, puntuación subescala positivo PANSS, puntuación subescala negativo PANSS y PANSS general (evidencia de muy baja calidad). En todas estas escalas, la olanzapina fue más eficaz que los antipsicóticos de primera generación (haloperidol, perfenazina) y los antipsicóticos de segunda generación (olanzapina, quetiapina, risperidona, ziprasidona) más eficaces que los antipsicóticos de primera generación (haloperidol, perfenazina) (evidencia de muy baja calidad). En la evaluación de la mejoría de los síntomas depresivos con la escala Ham-D en 28 pacientes ambulatorios tratados durante 14 semanas, no hubo diferencias entre la olanzapina y la risperidona (evidencia de baja calidad) (Akerele y Levin, 2007).

Respecto a las variables pragmáticas para los resultados de efectos secundarios, adherencia al tratamiento, abandono del tratamiento y reingreso hospitalario, no se encontraron diferencias significativas entre olanzapina y risperidona. En el resultado de abandono del tratamiento por cualquier motivo, se obtuvieron resultados similares en las comparaciones de olanzapina vs. perfenazina y antipsicóticos de segunda generación vs. perfenazina (evidencia de muy baja calidad).

\section{Recomendaciones}

- En los pacientes con esquizofrenia y policonsumo, el uso de antipsicóticos de segunda generación sobre 
Tabla 5. Estudios en esquizofrenia y varios trastornos por uso de sustancias.

\begin{tabular}{|c|c|c|c|c|c|c|c|}
\hline Autor & Diseño & Intervención & Pacientes & $\begin{array}{l}\operatorname{Exp}(n) / \\
\operatorname{Comp}(n)\end{array}$ & Seguimiento & $\begin{array}{c}\text { Variables de resultado } \\
\text { (clínicas, de consumo y } \\
\text { pragmáticas) }\end{array}$ & $\begin{array}{l}\text { Limitaciones } \\
\text { Sesgos }\end{array}$ \\
\hline $\begin{array}{l}\text { Akerele } \\
2007\end{array}$ & $\begin{array}{l}\text { ECA, } \\
\text { Doble-Ciego, } \\
\text { Ambulatorio }\end{array}$ & $\begin{array}{l}\text { 1. OLZ 5-20mg/d } \\
\text { 2. RIS } 3-9 \mathrm{mg} / \mathrm{d}\end{array}$ & $\begin{array}{l}\text { Esquizofrenia o Tr } \\
\text { Esquizoafectivo } \\
\text { + TUS (cocaína, } \\
\text { cannabis). } \\
\text { SCID. }\end{array}$ & $14 / 14$ & $14 \mathrm{sem}$ & $\begin{array}{l}\text { - HAM-D. PANSS positiva, } \\
\text { PANSS negativa. } \\
\text { - Test de Orina, Craving. } \\
\text { - Efectos secundarios: } \\
\text { AIMS, SAS. Conformidad. }\end{array}$ & $\begin{array}{l}\text { Muestra pequeña. Seguimiento } \\
\text { corto. Mayoritariamente } \\
\text { hombres ( } 89 \%) \text {. Posible sesgo } \\
\text { de publicación selectiva. No se } \\
\text { recogieron datos de consumo de } \\
\text { otras sustancias. Financiado por Eli } \\
\text { Lilly. Abandonos de seguimiento: El } \\
57,1 \% \text { finalizó el estudio (OLZ n=6; } \\
\text { RIS } n=10) \text {. }\end{array}$ \\
\hline $\begin{array}{l}\text { Green } \\
2004\end{array}$ & $\begin{array}{l}\text { ECA, } \\
\text { Doble-Ciego }\end{array}$ & $\begin{array}{l}\text { 1. OLZ } 5-20 \mathrm{mg} / \mathrm{d} \\
\text { 2. HAL } 2-20 \mathrm{mg} / \mathrm{d}\end{array}$ & $\begin{array}{c}\text { Primer episodio } \\
\text { psicótico } \\
\text { (Esquizofrenia, } \\
\text { TEA, } \\
\text { Esquizofreniforme) } \\
\text { + TUS (cannabis, } \\
\text { alcohol). } \\
\text { DSM-IV, SCID. }\end{array}$ & $131 / 131$ & 12 sem & $\begin{array}{l}\text { - PANSS, CGI. } \\
\text { Respuesta a } \\
\text { tratamiento. }\end{array}$ & $\begin{array}{l}\text { Criterio exclusión: dependencia de } \\
\text { sustancias en el último mes. Lilly } \\
\text { Research Laboratoies participó } \\
\text { de forma indirecta/directa en el } \\
\text { estudio. }\end{array}$ \\
\hline $\begin{array}{l}\text { Swartz } \\
2008\end{array}$ & $\begin{array}{l}\text { ECA, } \\
\text { Doble-Ciego, } \\
\text { Multicéntrico } \\
\text { Hospitalizado } \\
\text { +ambulatorio }\end{array}$ & $\begin{array}{l}\text { 1. OLZ } 7,5-30 \mathrm{mg} / \mathrm{d} \\
\text { 2. PRZ } 8-32 \mathrm{mg} / \mathrm{d} \\
\text { 3. QUE } 200-800 \mathrm{mg} / \mathrm{d} \\
\text { 4. RIS } 1,5-6 \mathrm{mg} / \mathrm{d} \\
\text { 5. ZPR } 40-160 \mathrm{mg} / \mathrm{d}\end{array}$ & $\begin{array}{l}\text { Esquizofrenia + } \\
\text { TUS o no-TUS } \\
\text { SCID. }\end{array}$ & $\begin{array}{c}(\mathrm{N}= \\
1432) . \\
\text { OLZ 142/ } \\
\text { PRZ 124/ } \\
\text { QUE 137/ } \\
\text { RIS 157/ } \\
\text { ZPS 83 }\end{array}$ & 18 meses & $\begin{array}{l}\text { - CGI-S, PANSS. } \\
\text { - Tasa de discontinuación. } \\
\text { Hospitalización. }\end{array}$ & $\begin{array}{l}\text { La dosis de medicación fue flexible } \\
\text { y basadas en el juicio clínico. La } \\
\text { adherencia fue monitorizada a } \\
\text { través del recuento del número } \\
\text { de pastillas. Posible sesgo de } \\
\text { selección: "los pacientes con } \\
\text { discinesia tardía concurrente } \\
\text { ( } \mathrm{n}=231 \text { ), entraron en un esquema } \\
\text { de aleatorización que los prevenía } \\
\text { de entrar en la rama de tratamiento } \\
\text { con PER". }\end{array}$ \\
\hline
\end{tabular}

Nota. AIMS: Abnormal Involuntary Movement Scale; CGI: Clinical Global Impression, Impresión Clínica Global; ECA: Ensayo clinico aleatorizado; HAL: Haloperidol; HAM-D: Hamilton Depression Rating Scale; OLZ: Olanzapina; PANSS: Positive and Negative Syndrome Scale; PRZ: Perfenazina; QUE: Quetiapina; RIS: Risperidona; SAS: Simpson Angus Scale; SCID: Structured Clinical Interview for DSM-IV; TEA: Trastorno Esquizoafectivo; TUS: Trastorno por Uso de Sustancias; ZPR: Ziprasidona.

antipsicóticos de primera generación y de olanzapina sobre otros antipsicóticos de segunda generación se recomienda para mejorar los síntomas psicóticos (recomendación moderada/débil).

\section{Tratamiento psicológico}

Pregunta 12 de PICO. ¿Es eficaz la administración de tratamiento psicológico para mejorar los síntomas de la esquizofrenia, reducir el consumo de sustancias o mejorar las variables pragmáticas y de funcionalidad en pacientes con esquizofrenia y policonsumo de drogas?

Un metanálisis de 32 ECA con 3165 participantes evaluó los efectos de las intervenciones psicosociales para la reducción del consumo de sustancias en personas con una enfermedad mental grave en comparación con la atención estándar (Hunt et al., 2013). No se observaron beneficios en la mejoría de los síntomas psicóticos medidos con escalas clínicas para las comparaciones del tratamiento habitual con la terapia cognitivo-conductual (TCC), TCC/ entrevista motivacional (EM) y solo la EM (evidencia de muy baja calidad). Para el resultado de interés de la disminución del consumo de drogas, incluido el consumo de alcohol, cannabis y sustancias en diferentes intervalos de tiempo (6, 12 y 36 meses), no se documentaron diferencias entre la atención habitual y el tratamiento integral, TCC, TCC/EM y EM (evidencia de baja o muy baja calidad). En la abstinencia de alcohol durante seis meses, un ECA mostró diferencias significativas a favor de la EM (evidencia de muy baja calidad). No se encontraron diferencias en las intervenciones vs. el tratamiento habitual en otros resultados, incluida la reducción de las hospitalizaciones o la funcionalidad global.

\section{Recomendaciones}

- En pacientes con esquizofrenia y trastorno por abuso de sustancias concurrente, no se puede hacer ninguna recomendación con respecto a la intervención psicológica más adecuada para mejorar los síntomas psicóticos, disminuir el uso de sustancias o mejorar la funcionalidad.

\section{Conclusiones}

Esta revisión proporciona una descripción general de la eficacia del tratamiento farmacológico y psicológico para los pacientes con esquizofrenia y un TUS comórbido. Es habitual que se presenten adultos y jóvenes con esquizofrenia y TUS comórbido para tratamiento en la práctica clínica. Una de las principales fortalezas de nuestra revisión consiste en la estricta selección de ECA. Sin embargo, 
aunque se revelaron conocimientos útiles sobre la eficacia del tratamiento ambulatorio de una patología dual, el escaso número de estudios incluidos, la evidencia claramente de muy baja calidad y los tamaños muestrales muy pequeños ilustran la necesidad de investigación adicional de alta calidad. Por tanto, son limitados los datos disponibles de tratamiento que muestran prácticas de tratamiento preferenciales respecto al uso de intervenciones específicas farmacológicas o psicológicas para las personas con esquizofrenia y los trastornos por uso de sustancias comórbidos.

Nuestros resultados sugieren que

1.En pacientes con esquizofrenia y consumo de cannabis, no es posible recomendar un fármaco antipsicótico sobre otro (entre olanzapina, risperidona o haloperidol) para mejorar los síntomas psicóticos, reducir el consumo de cannabis o mejorar las variables pragmáticas (recomendación débil). No se puede recomendar la clozapina para reducir el consumo de cannabis (recomendación débil). Se recomienda imipramina adyuvante para mejorar los síntomas afectivos (recomendación débil).

2. En pacientes con esquizofrenia y consumo de cocaína, recomendamos haloperidol sobre olanzapina para reducir el craving (recomendación moderada), sin embargo, se recomienda olanzapina sobre haloperidol para mejorar los efectos secundarios motores en estos pacientes (recomendación moderada). No se puede recomendar el uso de agonistas dopaminérgicos (mazindol) para mejorar los síntomas psicóticos, reducir el consumo de cocaína o el craving de cocaína o mejorar las variables pragmáticas (recomendación débil).

3. En pacientes con esquizofrenia y trastorno por consumo de alcohol, no hay evidencia suficiente para hacer recomendación alguna sobre el uso de antipsicóticos para mejorar los síntomas psicóticos, reducir el consumo de alcohol y/o craving de alcohol o mejorar las variables pragmáticas (recomendación débil). Sin embargo, mientras que se recomienda la naltrexona para reducir el consumo de alcohol (en términos de reducir el craving de alcohol) (recomendación débil), no hay evidencia suficiente para hacer recomendación alguna sobre el uso acamprosato adyuvante para mejorar los síntomas psicóticos, reducir el consumo de alcohol o mejorar las variables pragmáticas (recomendación débil).

4. En los pacientes con esquizofrenia y dependencia a la nicotina, no podemos recomendar el uso de bupropión para reducir los síntomas psicóticos (recomendación débil). Se recomienda bupropión o vareniclina adyuvante para reducir el uso de nicotina y la abstinencia de nicotina (recomendación fuerte/moderada).
5. En pacientes con esquizofrenia y policonsumo, se recomienda el uso de antipsicóticos de segunda generación frente a los de primera generación y de olanzapina frente a otros antipsicóticos de segunda generación para mejorar los síntomas psicóticos (recomendación moderada/débil).

6. En pacientes con esquizofrenia y trastorno por abuso de sustancias concurrente, no se puede hacer ninguna recomendación con respecto a la intervención psicológica más adecuada para mejorar los síntomas psicóticos, disminuir el uso de sustancias o mejorar la funcionalidad.

Las mejores prácticas incluyen tratamientos integrados para psicosis y uso de sustancias, enfatizando la inclusión en el tratamiento, la evaluación continua de los patrones de uso de sustancias y la atención coordinada que intenta alinear las necesidades de tratamiento con la gravedad de ambos trastornos y la etapa de cambio (De Witte, Crunelle, Sabbe, Moggi y Dom, 2014). Aunque el tratamiento de las personas con esquizofrenia y un diagnóstico comórbido de trastorno por uso de sustancias conlleva desafíos, los datos de los resultados muestran que el tratamiento es beneficioso y que existe un optimismo significativo para mejoras potencialmente mayores cuando se detiene el consumo de sustancias (Crockford y Addington, 2017).

\section{Reconocimientos}

Este estudio ha recibido apoyo financiero de: Servicio Gallego de Salud, SERGAS; Sociedad Española de Psiquiatría Biológica (SEPB), Pla Director de Salut Mental i Adiccions, Barcelona.

\section{Conflicto de intereses}

Los autores declaran la inexistencia de conflicto de interés alguno en relación a este artículo.

\section{Referencias}

Addy, P. H., Radhakrishnan, R., Cortes, J. A. y D’Souza, D. C. (2012). Comorbid alcohol, cannabis, and cocaine use disorders in schizophrenia: Epidemiology, consequences, mechanisms, and treatment. FOCUS: The Journal of Lifelong Learning in Psychiatry, 10, 140-153. doi:10.1176/ appi.focus.10.2.140.

Akbarpour, F., Rezaei, O., Khodaie-Ardakani, M. R., Sheikhvatan, M., Goodarzi, H. y Dolatshahi, B. (2010). A double-blind placebo controlled trial of bupropion for smoking abstinence and cognition improvement in schizophrenia. Minerva Psichiatrica, 51, 263-269.

Akerele, E. y Levin, F. R. (2007). Comparison of olanzapine to risperidone in substance-abusing individuals with 
schizophrenia. The American Journal on Addictions, 16, 260-268. doi:10.1080/10550490701389658.

Arranz, B., Garriga, M., García-Rizo, C. y San, L. (2018). Clozapine use in patients with schizophrenia and a comorbid substance use disorder: A systematic review. European Neuropsychopharmacology, 28, 227-242. doi:10.1016/j. euroneuro.2017.12.006.

Baker, A. L., Thornton, L. K., Hiles, S., Hides, L. y Lubman, D. I. (2012). Psychological interventions for alcohol misuse among people with co-occurring depression or anxiety disorders: A systematic review. Journal of Affective Disorders, 139, 217-229. doi:10.1016/j.jad.2011.08.004.

Berk, M., Brook, S. y Trandafir, A. I. (1999). A comparison of olanzapine with haloperidol in cannabis-induced psychotic disorder: A double-blind randomized controlled trial. International Clinical Psychopharmacology, 14, 177-180.

Bloch, B., Reshef, A., Cohen, T., Tafla, A., Gathas, S., Israel, S.,... Ebstein, R. P. (2010). Preliminary effects of bupropion and the promoter region (HTTLPR) serotonin transporter (SLC6A4) polymorphism on smoking behavior in schizophrenia. Psychiatry Research, 175, 38-42. doi:10.1016/j.psychres.2008.12.015.

Brunette, M. F., Dawson, R., O'Keefe, C. D., Narasimhan, M., Noordsy, D. L., Wojcik, J. y Green, A. I. (2011). A randomized trial of clozapine vs. other antipsychotics for cannabis use disorder in patients with schizophrenia. Journal of Dual Diagnosis, 7, 50-63. doi:10.1080/155 04263.2011 .570118 .

Crockford, D. y Addington, D. (2017). Canadian Schizophrenia Guidelines: Schizophrenia and other psychotic disorders with coexisting substance use disorders. Canadian Journal of Psychiatry, 62, 624-634. doi:10.1177/0706743717720196.

De Witte, N. J., Crunelle, C. L., Sabbe, B., Moggi, F. y Dom, G. (2014). Treatment for outpatients with comorbid schizophrenia and substance use disorders: A review. European Addiction Research, 20, 105-114. doi:10.1159/000355267.

Drake, R. E., Xie, H., McHugo, G. J. y Green, A. I. (2000). The effects of clozapine on alcohol and drug use disorders among patients with schizophrenia. Schizophrenia Bulletin, 26, 441-449.

Evins, A. E., Cather, C., Culhane, M. A., Birnbaum, A., Horowitz, J., Hsieh, E.,... Goff, D. C. (2007). A 12-week double-blind, placebo-controlled study of bupropion SR added to high-dose dual nicotine replacement therapy for smoking cessation or reduction in schizophrenia. Journal of Clinical Psychopharmacology, 27, 380-386. doi:10.1097/01.jcp.0b013e3180ca86fa.

Evins, A. E., Cather, C., Deckersbach, T., Freudenreich, O., Culhane, M. A., Olm-Shipman, C. M., Henderson, D. C.,... Rigotti, N. A. (2005). A double-blind placebo-controlled trial of bupropion sustained-release for smoking cessation in schizophrenia. Journal of Clinical Psychopharmacology, 25, 218-225. doi:10.1097/01. jcp.0000162802.54076.18.

Evins, A. E., Mays, V. K., Rigotti, N. A., Tisdale, T., Cather, C. y Goff, D. C. (2001). A pilot trial of bupropion added to cognitive behavioral therapy for smoking cessation in schizophrenia. Nicotine y Tobacco Research, 3, 397-403. doi:10.1080/14622200110073920.

Fonseca-Pedrero, E., Lucas-Molina, B., Pérez-Albéniz, A., Inchausti, F. y Ortuño-Sierra, J. (2020). Psychotic-like experiences and cannabis use in adolescents from the general population. Adicciones, 32, 41-51. doi:10.20882/ adicciones.1149.

García, L., Gomar, J. J., García-Portilla, M. P. y Bobes, J. (2019). Cannabis use and cognitive impairment in schizophrenia and first-episode psychosis. Adicciones, 31, 89-94. doi:10.20882/adicciones.1328.

George, T., Vessicchio, J., Sacco, K., Weinberger, A., Dudas, M., Allen, T.,... Jatlow, P. I. (2008). A placebo-controlled trial of bupropion combined with nicotine patch for smoking cessation in schizophrenia. Biological Psychiatry, 63, 1092-1096. doi:10.1016/j.biopsych.2007.11.002.

George, T., Vessicchio, J., Termine, A., Bregartner, T., Feingold, A., Rounsaville, B. y Kosten, T. (2002). A placebo controlled trial of bupropion for smoking cessation in schizophrenia. Biological Psychiatry, 52, 53-61. doi:10.1016/S0006-3223(02)01339-2.

Gopalakrishna, G., Langendam, M. W., Scholten, R. J., Bossuyt, P. M. y Leeflang, M. M. (2013). Guidelines for guideline developers: A systematic review of grading systems for medical tests. Implementation Science, 8, 78. doi:10.1186/1748-5908-8-78.

Green, A., Noordsy, D. L., Brunette, M. F. y O'Keefe, C. (2008). Substance abuse and schizophrenia: Pharmacotherapeutic intervention. Journal of Substance Abuse Treatment, 34, 61-71. doi:10.1016/j.jsat.2007.01.008.

Green, A., Tohen, M. F., Hamer, R. M., Strakowski, S. M., Lieberman, J. A., Glick, I.,... Scott Clark, W. (2004). First episode schizophrenia-related psychosis and substance use disorders: Acute response to olanzapine and haloperidol. Schizophrenia Research, 66, 125-135. doi:10.1016/j. schres.2003.08.001.

Green, A., Zimmet, S. V, Strous, R. D. y Schildkraut, J. J. (1999). Clozapine for comorbid substance use disorder and schizophrenia: Do patients with schizophrenia have a reward-deficiency syndrome that can be ameliorated by clozapine? Harvard Review of Psychiatry, 6, 287-296.

Guyatt, G. H., Oxman, A. D., Kunz, R., Atkins, D., Brozek, J., Vist, G.,... Schünemann, H. J. (2011). GRADE guidelines: 2. Framing the question and deciding on important outcomes. Journal of Clinical Epidemiology, 64, 395-400. doi:10.1016/j.jclinepi.2010.09.012.

Hasan, A., Falkai, P., Wobrock, T., Lieberman, J., Glenthoj, B., Gattaz, W. F.,... Möller, H.-J. (2012). World Federa- 
tion of Societies of Biological Psychiatry (WFSBP) Guidelines for Biological Treatment of Schizophrenia, Part 1: Update 2012 on the acute treatment of schizophrenia and the management of treatment resistance. World Journal of Biological Psychiatry, 13, 318-378. doi:10.3109/ 15622975.2012.696143.

Hasan, A., Falkai, P., Wobrock, T., Lieberman, J., Glenthøj, B., Gattaz, W. F.,... Möller, H.-J. (2015). World Federation of Societies of Biological Psychiatry (WFSBP) Guidelines for Biological Treatment of Schizophrenia Part 3: Update 2015 Management of special circumstances: Depression, suicidality, substance use disorders and pregnancy and lactation. The World Journal of Biological Psychiatry, 16, 142-170. doi:10.3109/15622975.2015.10 09163.

Heiberg, I. H., Jacobsen, B. K., Nesvåg, R., Bramness, J. G., Reichborn-Kjennerud, T., Næss, Ø., ... Høye, A. (2018). Total and cause-specific standardized mortality ratios in patients with schizophrenia and/or substance use disorder. PLoS One, 13, e0202028. doi:10.1371/journal. pone.0202028.

Hunt, G. E., Large, M. M., Cleary, M., Lai, H. M. X. y Saunders, J. B. (2018). Prevalence of comorbid substance use in schizophrenia spectrum disorders in community and clinical settings, 1990-2017: Systematic review and meta-analysis. Drug and Alcohol Dependence, 191, 234-258. doi:10.1016/j.drugalcdep.2018.07.011.

Hunt, G. E., Siegfried, N., Morley, K., Sitharthan, T. y Cleary, M. (2013). Psychosocial interventions for people with both severe mental illness and substance misuse. The Cochrane Database of Systematic Reviews, 10, CD001088. doi:10.1002/14651858.CD001088.pub3.

Kavanagh, D. J., Mcgrath, J., Saunders, J. B., Dore, G. y Clark, D. (2002). Substance misuse in patients with schizophrenia: Epidemiology and management. Drugs, 62, 743-755.

Kishi, T. y Iwata, N. (2015). Varenicline for smoking cessation in people with schizophrenia: Systematic review and meta-analysis. European Archives of Psychiatry and Clinical Neuroscience, 265, 259-268. doi:10.1007/s00406014-0551-3.

Krause, M., Huhn, M., Schneider-Thoma, J., Bighelli, I., Gutsmiedl, K. y Leucht, S. (2019). Efficacy, acceptability and tolerability of antipsychotics in patients with schizophrenia and comorbid substance use. A systematic review and meta-analysis. European Neuropsychopharmacology, 29, 32-45. doi:10.1016/j.euroneuro.2018.11.1105.

Large, M., Mullin, K., Gupta, P., Harris, A. y Nielssen, O. (2014). Systematic meta-analysis of outcomes associated with psychosis and co-morbid substance use. The Australian and New Zealand Journal of Psychiatry, 48, 418-432. doi:10.1177/0004867414525838.

Makarski, J. y Brouwers, M. C. (2014). The AGREE Enterprise: A decade of advancing clinical practice guideli- nes. Implementation Science, 9, 103. doi:10.1186/s13012014-0103-2.

Margolese, H. C., Malchy, L., Negrete, J. C., Tempier, R. y Gill, K. (2004). Drug and alcohol use among patients with schizophrenia and related psychoses: Levels and consequences. Schizophrenia Research, 67, 157-166. doi:10.1016/S0920-9964(02)00523-6.

Matali, J. L., Andión, O., Pardo, M., Iniesta, R., Serrano, E. y San, L. (2016). Adolescents and dual diagnosis in a psychiatric emergency service. Adicciones, 28, 71-79. doi:10.20882/adicciones. 783.

Mueser, K. T. y Gingerich, S. (2013). Treatment of co-occurring psychotic and substance use disorders. Social Work in Public Health, 28, 424-439. doi:10.1080/19371918.20 13.774676.

Murthy, P., Mahadevan, J. y Chand, P. K. (2019). Treatment of substance use disorders with co-occurring severe mental health disorders. Current Opinion in Psychiatry, 32, 293-299. doi:10.1097/YCO.0000000000000510.

Mustafa, R. A., Santesso, N., Brozek, J., Akl, E. A., Walter, S. D., Norman, G.,... Schünemann, H. J. (2013). The GRADE approach is reproducible in assessing the quality of evidence of quantitative evidence syntheses. Journal of Clinical Epidemiology, 66, 736-742. doi:10.1016/j. jclinepi.2013.02.004.

Nielsen, S. M., Toftdahl, N. G., Nordentoft, M. y Hjorthøj, C. (2017). Association between alcohol, cannabis, and other illicit substance abuse and risk of developing schizophrenia: A nationwide population based register study. Psychological Medicine, 47, 1668-1677. doi:10.1017/ S0033291717000162.

Pearsall, R., Smith, D. J. y Geddes, J. R. (2019). Pharmacological and behavioural interventions to promote smoking cessation in adults with schizophrenia and bipolar disorders: A systematic review and meta-analysis of randomised trials. BMJ Open, 9, 1-12. doi:10.1136/ bmjopen-2018-027389.

Perry, E. B., Gil, R., Miles, D., Brenner, L., Macdougall, L., Johnson, R.,... D’Souza, D. C. (2005). Mazindol augmentation of antipsychotic treatment for schizophrenic patients with comorbid cocaine abuse or dependence. Journal of Dual Diagnosis, 1, 37-47. doi:10.1300/ J374v01n01_04.

Petrakis, I. L., O’Malley, S., Rounsaville, B., Poling, J., McHugh-Strong, C. y Krystal, J. H. (2004). Naltrexone augmentation of neuroleptic treatment in alcohol abusing patients with schizophrenia. Psychopharmacology, 172, 291-297. doi:10.1007/s00213-003-1658-9.

Ralevski, E., O’Brien, E., Jane, J. S., Dean, E., Dwan, R. y Petrakis, I. (2011). Effects of acamprosate on cognition in a treatment study of patients with schizophrenia spectrum disorders and comorbid alcohol dependence. The Journal of Nervous and Mental Disease, 199, 499-505. doi:10.1097/NMD.0b013e3182214297. 
Regier, D. A., Farmer, M. E., Rae, D. S., Locke, B. Z., Keith, S. J., Judd, L. L. y Goodwin, F. K. (1990). Comorbidity of mental disorders with alcohol and other drug abuse. Results from the Epidemiologic Catchment Area (ECA) Study. JAMA, 264, 2511-2518.

Sayers, S. L., Campbell, E. C., Kondrich, J., Mann, S. C., Cornish, J., O’ Brien, C. y Caroff, S. N. (2005). Cocaine abuse in schizophrenic patients treated with olanzapine versus haloperidol. The Journal of Nervous and Mental Disease, 193, 379-386. doi:10.1097/01.nmd.0000165089.14736. bf.

Schünemann, H. J., Oxman, A. D., Brozek, J., Glasziou, P., Jaeschke, R., Vist, G. E.,... Guyatt, G. H. (2008). Grading quality of evidence and strength of recommendations for diagnostic tests and strategies. BMJ, 336, 1106-1110. doi:10.1136/bmj.39500.677199.AE.

Sevy, S., Robinson, D. G., Sunday, S., Napolitano, B., Miller, R., McCormack, J. y Kane, J. (2011). Olanzapine vs. risperidone in patients with first-episode schizophrenia and a lifetime history of cannabis use disorders: 16-week clinical and substance use outcomes. Psychiatry Research, 188, 310-314. doi:10.1016/j.psychres.2011.05.001.

Siris, S. G., Bermanzohn, P. C., Mason, S. E., Rifkin, A. y Alvir, J. M. (1992). Adjunctive imipramine for dysphoric schizophrenic patients with past histories of cannabis abuse. Progress in Neuro-Psychopharmacology y Biological Psychiatry, 16, 539-547.

Smelson, D. A., Ziedonis, D., Williams, J., Losonczy, M. F., Williams, J., Steinberg, M. L. y Kaune, M. (2006). The efficacy of olanzapine for decreasing cue-elicited craving in individuals with schizophrenia and cocaine dependence. Journal of Clinical Psychopharmacology, 26, 9-12. doi:10.1097/01.jcp.0000194624.07611.5e.

Swartz, M. S., Wagner, H. R., Swanson, J. W., Stroup, T. S., McEvoy, J. P., Reimherr, F.,... Lieberman, J. A. (2008). The effectiveness of antipsychotic medications in patients who use or avoid illicit substances: Results from the CATIE study. Schizophrenia Research, 100, 39-52. doi:10.1016/j.schres.2007.11.034.

Temmingh, H. S., Williams, T., Siegfried, N. y Stein, D. J. (2018). Risperidone versus other antipsychotics for people with severe mental illness and co-occurring substance misuse. The Cochrane Database of Systematic Reviews, 1, CD011057. doi:10.1002/14651858.CD011057.pub2.

Thornton, L. K., Baker, A. L., Lewin, T. J., Kay-Lambkin, F. J., Kavanagh, D., Richmond, R.,... Johnson, M. P. (2012). Reasons for substance use among people with mental disorders. Addictive Behaviors, 37, 427-434. doi:10.1016/j. addbeh.2011.11.039.

Van Nimwegen, L. J., de Haan, L., van Beveren, N. J., van der Helm, M., van den Brink, W. y Linszen, D. (2008). Effect of olanzapine and risperidone on subjective well-being and craving for cannabis in patients with schizophrenia or related disorders: A double-blind rando- mized controlled trial. Canadian Journal of Psychiatry, 53, 400-405.

Weiner, E., Ball, M. P., Buchholz, A. S., Gold, J. M., Evins, A. E., McMahon, R. P. y Buchanan, R. W. (2012). Bupropion sustained release added to group support for smoking cessation in schizophrenia. The Journal of Clinical Psychiatry, 73, 95-102. doi:10.4088/JCP.10m06143gre.

Weiner, E., Buchholz, A., Coffay, A., Liu, F., McMahon, R. P., Buchanan, R. W. y Kelly, D. L. (2011). Varenicline for smoking cessation in people with schizophrenia: A double blind randomized pilot study. Schizophrenia Research, 129, 94-95. doi:10.1016/j.schres.2011.02.003.

Williams, J. M., Anthenelli, R. M., Morris, C. D., Treadow, J., Thompson, J. R., Yunis, C. y George, T. P. (2012). A randomized, double-blind, placebo-controlled study evaluating the safety and efficacy of varenicline for smoking cessation in patients with schizophrenia or schizoaffective disorder. The Journal of Clinical Psychiatry, 73, 654-660. doi:10.4088/JCP.11m07522.

Wilson, R. P. y Bhattacharyya, S. (2016). Antipsychotic efficacy in psychosis with co-morbid cannabis misuse: A systematic review. Journal of Psychopharmacology, 30, 99-111. doi:10.1177/0269881115612237.

Wobrock, T. y Soyka, M. (2008). Pharmacotherapy of schizophrenia with comorbid substance use disorder-reviewing the evidence and clinical recommendations. Progress in Neuro-Psychopharmacology y Biological Psychiatry, 32, 1375-1385. doi:10.1016/j.pnpbp.2008.02.008. 
